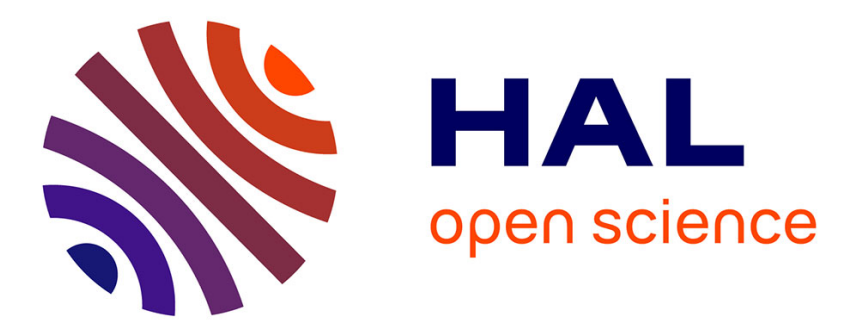

\title{
Dirac points emerging from flat bands in Lieb-kagome lattices
}

Jean-Noël Fuchs, Lih-King Lim, Frédéric Piéchon, Gilles Montambaux

\section{To cite this version:}

Jean-Noël Fuchs, Lih-King Lim, Frédéric Piéchon, Gilles Montambaux. Dirac points emerging from flat bands in Lieb-kagome lattices. Physical Review B, 2020, 101 (4), 10.1103/physrevb.101.045131. hal-02988788

\section{HAL Id: hal-02988788 \\ https://hal.science/hal-02988788}

Submitted on 4 Nov 2020

HAL is a multi-disciplinary open access archive for the deposit and dissemination of scientific research documents, whether they are published or not. The documents may come from teaching and research institutions in France or abroad, or from public or private research centers.
L'archive ouverte pluridisciplinaire HAL, est destinée au dépôt et à la diffusion de documents scientifiques de niveau recherche, publiés ou non, émanant des établissements d'enseignement et de recherche français ou étrangers, des laboratoires publics ou privés. 


\title{
Dirac points emerging from flat bands in Lieb-kagome lattices
}

\author{
Lih-King Lim,,${ }^{1}{ }^{*}$ Jean-Noël Fuchs, ${ }^{2}$ Frédéric Piéchon, ${ }^{3}$ and Gilles Montambaux ${ }^{3}$ \\ ${ }^{1}$ Zhejiang Institute of Modern Physics, Department of Physics, Zhejiang University, Hangzhou, Zhejiang 310027, People's Republic of China \\ ${ }^{2}$ Sorbonne Université, CNRS, Laboratoire de Physique Théorique de la Matière Condensée, LPTMC, F-75005 Paris, France \\ ${ }^{3}$ Laboratoire de Physique des Solides, CNRS, Université Paris-Sud, Université Paris-Saclay, F-91405 Orsay, France
}

(Received 8 July 2019; revised manuscript received 28 October 2019; published 28 January 2020)

\begin{abstract}
The energy spectra for the tight-binding models on the Lieb and kagome lattices both exhibit a flat band. We study a model which continuously interpolates between these two limits. The flat band located in the middle of the three-band spectrum for the Lieb lattice is distorted, generating two pairs of Dirac points. While the upper pair evolves into graphenelike Dirac cones in the kagome limit, the low-energy pair evolves until it merges, producing the band-bottom flat band. The topological characterization of the Dirac points is achieved by projecting the Hamiltonian on the two relevant bands in order to obtain an effective Dirac Hamiltonian. The low-energy pair of Dirac points is particularly interesting: when they emerge, they have opposite winding numbers, but as they merge, they have the same winding number. This apparent paradox is due to a continuous rotation of their states in pseudospin space, characterized by a winding vector. This simple, but quite rich model, suggests a way to a systematic characterization of two-band contact points in multiband systems.
\end{abstract}

DOI: 10.1103/PhysRevB.101.045131

\section{INTRODUCTION}

Multiband systems with flat band are of current interest thanks to their experimental realizations in a variety of systems [1-7]. The list includes cold atoms with optical lattices [1,3], and photonic [2,4,5] and polaritonic [6] systems. Typically, the systems are engineered to faithfully realize exotic tight-binding models with multiple orbitals or atoms per unit cell, as well as complex lattice geometries. In these artificial systems, direct imaging of localized states $[4,7,8]$ or the study of tunable interaction-induced effects [9] are few examples which show approaches hitherto not easily realizable in conventional condensed matter systems.

In this work, we study a connection between two twodimensional tight-binding models, on the Lieb lattice and on the kagome lattice (also known as the trihexagonal tiling), both featuring two dispersive bands and a flat band. On the one hand, the Lieb model [10-13] has a band contact between three bands among which a topological flat band [14] at zero energy [see Fig. 1(a), the tight-binding model is defined in Fig. 2(a)]. On the other hand, the kagome model $[15,16]$, chosen here with a square symmetry, features a quadratic band contact point [17-19] between its lower bands: a dispersive band and a flat band resulting from destructive interferences [14] [see Fig. 1(d)]. The real-space model is defined in Fig. 2(b). Apart from the flat band, the kagome energy spectrum is very similar to that of graphene with a pair of Dirac points between the upper bands [see Fig. 1(d)]. Both models have found physical realizations in the aforementioned systems [1-7]. There are also solid-state systems expected to behave as a kagome lattice for itinerant electrons (see, e.g., Refs. [20-22]).

*lihking@zju.edu.cn
By interpolating between the two lattice models, we define the Lieb-kagome model [see Fig. 2(c)]. It displays a smooth crossover of the flat band from the middle of the spectrum to the lowest energy. This model was already briefly introduced and its energy spectrum discussed by Asano and Hotta (see Fig. 7 in Ref. [23]). Of particular interest here is the continuous deformation of the flat band without gap opening when deviating from the two limits. This deformation is accompanied by the creation of pairs of Dirac points, i.e., linear band contact points between two bands.

Very recently, Jiang et al. [24] have considered the Liebkagome model in the presence of a time-reversal breaking term (related to intrinsic spin-orbit coupling) that gaps the energy spectrum. The topology of the isolated bands was then characterized by computing the corresponding Chern numbers, which obviously depends on the way the gap is opened. In contrast, in this paper, we do not gap the energy spectrum and instead characterize the band contact
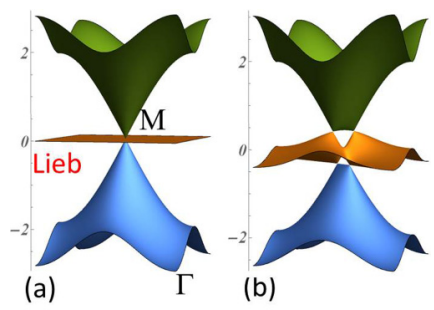

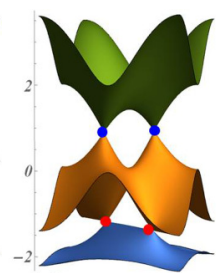

(c)

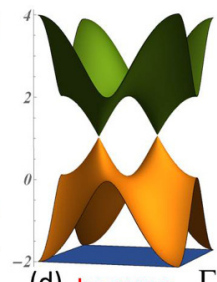

(d) kagome $\Gamma$
FIG. 1. Energy band spectrum of the Lieb-kagome model in the Brillouin zone centered on the $M$ point [see Fig. 3(a)]. (a) Lieb model $(t=0)$; (b) $t=0.2$; (c) $t=0.7$; (d) kagome model $(t=1)$ in a square version. One pair of Dirac points is, respectively, formed in the upper two (blue dots) and lower two bands (red dots) away from the two limits. 
points as topological defects by computing the associated charge.

On the one hand, slightly deforming the Lieb lattice, two pairs of Dirac points are generated from the three-band contact at the $M=(\pi, \pi)$ point of the first Brillouin zone (BZ) [see Figs. 1(a) and 1(b)]. One pair is between the lower two bands and will evolve in the flat band of the kagome limit. The other pair (between the upper two bands) will separate and turn into the Dirac points of the kagome lattice. As we will see below, near the $M$ point, each pair has opposite winding numbers $(+-)$. On the other hand, deforming the kagome lattice, a single pair of Dirac points emerges from the quadratic flat band contact at the $\Gamma$ point between the two lower bands [see Figs. 1(f) and 1(e)]. The latter has a +2 winding number that produces a pair of Dirac points with identical winding numbers $(++)$.

The evolution of the lower two bands then raises the question of how the two distinct fusion scenarios of Dirac points can be smoothly connected, namely, a $(+-)$ pair of Dirac points emerging at $M$ evolving into a $(++)$ pair merging at $\Gamma$. Actually, a similar phenomenon has been studied by us in a two-band model, the staggered Mielke lattice [25]. While the integer-valued winding number characterizes the circulation of the Dirac spinor on the great circle of the Bloch sphere, the great circle itself is not fixed generally: it evolves according to the Hamiltonian parameters. We have shown that, in order to describe properly a pair of Dirac points and their merging, the orientation of the great circle around each Dirac point has to be specified by a vector perpendicular to the plane of the great circle, that we named the winding vector. Then, during the smooth evolution between the two merging scenarios, the relative direction of winding vectors continuously evolves from a parallel to an antiparallel situation.

In the three-band Lieb-kagome model, the mechanism of distortion and restoration of the flat band implies the generation of Dirac pairs which follows different merging scenarios. The winding vector description a priori applies only in a two-band system. One of our main tasks is thus to find a consistent basis to represent the Lieb-kagome Hamiltonian, local in $\mathbf{k}$ space, from which effective two-band descriptions for the contact points can be derived.

There is a deep connection with the work by Ahn et al. on the topological characterization of real band structures [26]. This is best seen by working in a basis in which the Bloch Hamiltonian of the Lieb-kagome model is real. We detail this comparison in Appendix D.

The paper is organized as follow. In Sec. II, we define the model and give a brief summary of the universal Hamiltonians of Dirac points merging/emergence. In Secs. III and IV, we construct the smooth basis with which effective two-band Hamiltonians can be derived. Then, we determine the evolution of the winding vectors. In Sec. V, we give a global picture of the evolving winding vector and follow with conclusions. In the Appendices $\mathrm{A}$ and $\mathrm{B}$, we give details on several computations. We also generalize the Liebkagome model in Appendix C. In Appendix D, we discuss the Lieb-kagome model in an alternative representation in which the Bloch Hamiltonian is real and make contact with Ref. [26].
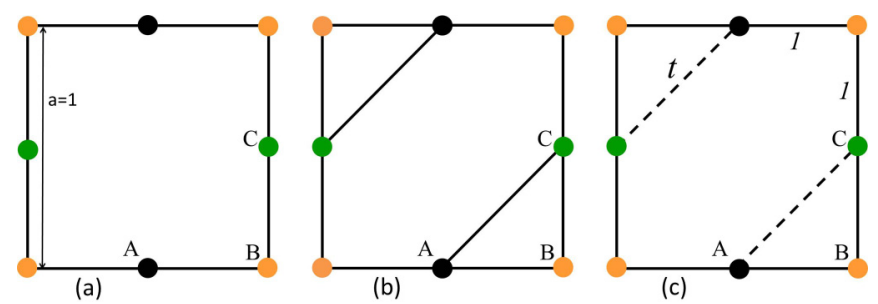

FIG. 2. Unit cell of (a) the Lieb lattice (full lines represent unit hopping amplitudes); (b) the kagome lattice in a square version; (c) the interpolating Lieb-kagome lattice (dashed lines correspond to hopping amplitudes $0 \leqslant t \leqslant 1$ ). The three sites in the unit cell are $\mathrm{A}, \mathrm{B}$, and $\mathrm{C}$.

\section{LIEB-KAGOME LATTICE AND CONTACT POINTS}

\section{A. Tight-binding model}

The Lieb-kagome tight-binding model is schematically shown in Fig. 2(c), where the full bonds indicate hopping amplitudes of fixed magnitude (which we take as energy unit) and the dashed bonds correspond to variable hopping of strength $t$. The unit cell contains three orbitals at sites A, B, and C, which results in three energy bands. In this work, we are interested in the evolution of the band structure for the parameter range $0 \leqslant t \leqslant 1$. For any $t$, this model has time-reversal symmetry, inversion symmetry [with center either on the B sites or in the middle of the unit cell shown in Fig. 2(c)], and it also possesses mirror symmetries with respect to reflections through the diagonal (d) and antidiagonal (a) lines passing through B sites of the unit cell. Two well-known limits of the above model are as follows:

(1) The Lieb lattice $t=0$. It is a regular square lattice with $\mathrm{B}$ sites forming the Bravais lattice and additional sites (A and $\mathrm{C}$ sites) located at the center in-between all the nearest-neighbor B sites [Fig. 2(a)]. In addition to the above symmetries, it is also bipartite (i.e., it has a chiral sublattice symmetry) and it possesses mirror symmetries along the $x$ axis and along the $y$ axis. Since it is bipartite, its spectrum respects the energy inversion $E \rightarrow-E$ symmetry and hence the middle band is necessarily a zero-energy flat band. According to the classification of Aoki et al., this flat band is of topological origin [14]. For example, its energy is not affected by a perpendicular magnetic field [14]. Another example of a topological flat band is found in the dice lattice [27].

(2) The kagome lattice $t=1$. Here it is deformed from the usual kagome to have a square symmetry but it has the same lattice connectivity. The kagome lattice belongs to a family of frustrated tight-binding models constructed on line graphs of bipartite parent lattices, of which the Mielke (checkerboard) lattice is another instance [28-30]. These models display a flat band at the minimum (or maximum) energy which is in contact with a dispersive band. The electron localization leading to the flat band is due to a destructive interference, which is affected by a perpendicular magnetic field [14].

We choose to write the Bloch Hamiltonian in the form $H(\mathbf{k})=e^{-i \mathbf{k} \cdot \mathbf{R}} H e^{i \mathbf{k} \cdot \mathbf{R}}$ where $\mathbf{R}$ are the positions of the Bravais lattice sites (see Appendices A and D 2). In the momentumspace basis $\{|\mathbf{k}, A\rangle,|\mathbf{k}, B\rangle,|\mathbf{k}, C\rangle\}$, the Bloch Hamiltonian can 


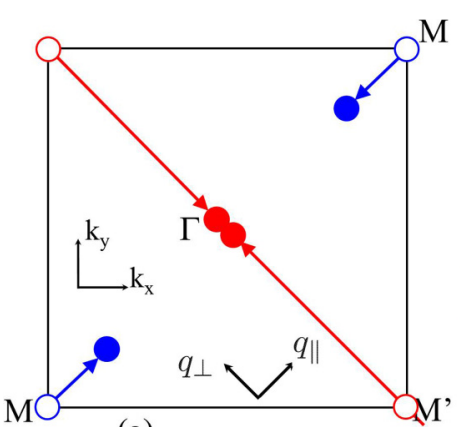

(a)

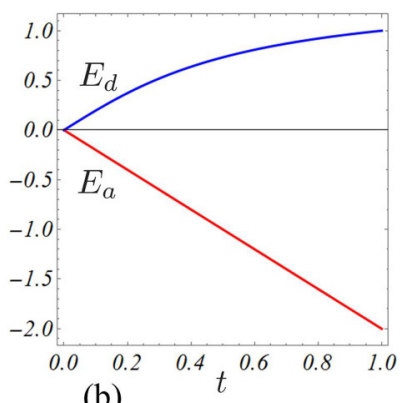

(b)
FIG. 3. (a) Evolution of the upper (lower) Dirac points in blue (red) in the first Brillouin zone (BZ). When $t$ increases from 0 to 1 , the lower pair (red) moves from the $M=(\pi, \pi)$ point to the $\Gamma=(0,0)$ point, while the upper pair (blue) moves from $M$ to $\xi \mathbf{K}=\xi(2 \pi / 3,2 \pi / 3)$ shown as filled blue disks ( $\xi= \pm 1$ is a valley index). (b) Evolution of the energy of the upper (blue) and lower (red) Dirac points between the Lieb $(t=0)$ and the kagome $(t=1)$ lattices.

be represented as

$$
H(\mathbf{k})=\left(\begin{array}{ccc}
0 & 1+e^{i k_{x}} & t\left(1+e^{i\left(k_{x}+k_{y}\right)}\right) \\
\cdots & 0 & 1+e^{i k_{y}} \\
\cdots & \cdots & 0
\end{array}\right)
$$

where we have chosen the lattice spacing $a=1$ [see Fig 2(a)].

In the following, we will use the following convention for Bloch Hamiltonians. Three-band Hamiltonians in the site basis will be denoted $H$, three-band Hamiltonians in another basis $\tilde{H}$, and two-band effective Hamiltonians $\mathcal{H}$.

\section{B. Energy spectrum}

The energy spectrum for $0 \leqslant t \leqslant 1$ is shown in Fig. 1, which displays three energy bands and remains gapless. The band-touching points and their characteristics depend on $t$.

For the Lieb lattice $(t=0)$ there is a triple band crossing at the $M$ point in momentum space $\left[k_{x}=k_{y}=\pi\right.$ and their equivalents, Fig. 3(a)]. It shows a linear contact point with a flat band in the middle, which is characteristic of a pseudospin-1 [12]. In deviating from the Lieb limit $(t>0)$, the flat band is distorted with the triple crossing point splitting into two pairs of Dirac points and particle-hole symmetry of the energy spectrum is lost. They are formed, respectively, in-between the lower and middle bands, and the middle and the upper bands, in two orthogonal directions in momentum space (see Fig. 4).

Moving away from the Lieb limit $t>0$, the distortion of the middle band gets more pronounced and the lower Dirac pair moves in the antidiagonal direction $\left(k_{x}=-k_{y}\right)$ toward the $\Gamma$ point of the $\mathrm{BZ}\left(k_{x}=k_{y}=0\right)$ [red arrows in Fig. 3(a)]. These Dirac cones are critically tilted such as to exhibit a zero-velocity line as seen on Figs. 1(c) and 5. In approaching the kagome limit $t \rightarrow 1$, the same Dirac points merge at the $\Gamma$ point forming a single contact point between a quadratic band and a flat band, thus restoring a flat lowest-energy band. The upper Dirac pair, on the other hand, moves along the diagonal direction $\left[k_{x}=k_{y}\right.$, blue arrows in Fig. 3(a)] toward a fixed position $\xi \mathbf{K}=\xi(2 \pi / 3,2 \pi / 3)$, where $\xi= \pm 1$ is a valley
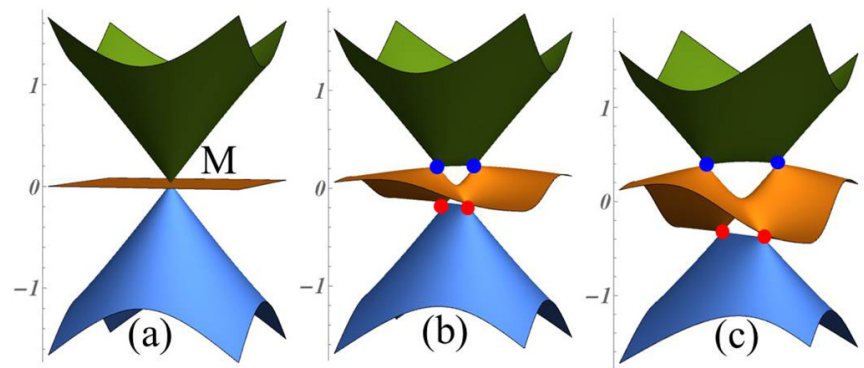

FIG. 4. Closeup energy spectrum at $M$ point showing the multiplets of Dirac points in the vicinity of the Lieb limit $t=$ $0(a), 0.1(b), 0.2(c)$.

index, while maintaining the Dirac cone structure throughout. The tilt of these Dirac cones diminishes with increasing $t$ and vanishes at $t=1$ [see Figs. 1(c) and 1(d)].

In summary, the crossover between flat bands happens by producing pairs $\left(\mathbf{k}_{D},-\mathbf{k}_{D}\right)$ of Dirac points. The lower pair evolves from the $M$ and the $\Gamma$ point. Its merging/emergence at time-reversal-invariant momenta (TRIM) resembles a situation we already encountered in the two-band model on a staggered Mielke lattice [25]. There, we provided a complete scenario of a $(+-)$ going into a $(++)$ Dirac pair. In the following subsection we briefly summarize the main results of Ref. [25].

\section{Characterization of contact points: $2 \times 2$ effective Hamiltonians}

A contact point between two bands is characterized by an effective $2 \times 2$ Hamiltonian. Around a single contact point $\mathbf{k}_{D}$, the local two-band Bloch Hamiltonian takes on a general form

$$
\mathcal{H}(\mathbf{q})=h_{\mu}(\mathbf{q}) \sigma_{\mu}+h_{v}(\mathbf{q}) \sigma_{v} \equiv \vec{h}(\mathbf{q}) \cdot \vec{\sigma}
$$

involving only two among three Pauli matrices $\sigma_{\mu}=\vec{u}_{\mu}\left(\mathbf{k}_{D}\right)$. $\vec{\sigma}$ and $\sigma_{v}=\vec{u}_{v}\left(\mathbf{k}_{D}\right) \cdot \vec{\sigma}$ with $\left|\vec{u}_{\mu, v}\right|=1, \vec{u}_{\mu} \cdot \vec{u}_{v}=0$ (e.g., $\mu, v=x, y)$, and $\vec{\sigma}=\left(\sigma_{x}, \sigma_{y}, \sigma_{z}\right)$. The winding number is the number of times the effective magnetic field $\vec{h}(\mathbf{q})$ winds on a great circle of the Bloch sphere in the $\left(\vec{u}_{\mu}, \vec{u}_{\nu}\right)$ plane when $\mathbf{q}$ encircles the contact point once in the trigonometric direction. For example, for a Dirac point one can have $\vec{h}(\mathbf{q})=$ $\left(v_{x} q_{x}, v_{y} q_{y}, 0\right)$ and the winding number is $\operatorname{sgn}\left(v_{x} v_{y}\right)$.

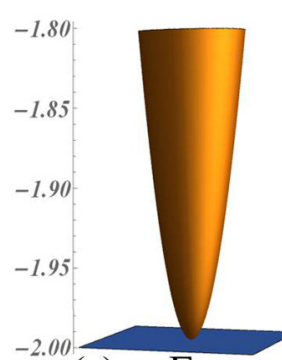

(a)

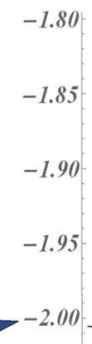

(b)

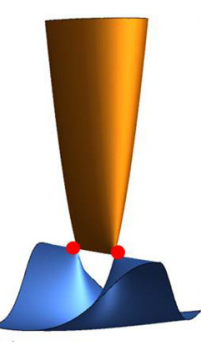

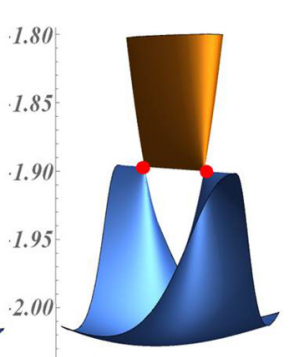

(c)
FIG. 5. Closeup energy spectrum at $\Gamma$ point showing the emergence of Dirac points from the kagome limit $t=1(a), 0.98(b), 0.95(c)$. 
Indeed, the stability of two-band contact points in two dimensions (2D) requires a symmetry protection (such as a chiral symmetry or inversion and time-reversal symmetries). The associated topological charge is the $\mathbb{Z}$ winding number that classifies maps from a loop $S^{1}$ encircling the contact point in reciprocal space to a great circle $S^{1}$ of the Bloch sphere associated to the $2 \times 2$ Bloch Hamiltonian $\mathcal{H}(\mathbf{q})$. In the absence of such a symmetry, band contact points are unstable defects in 2D [31].

To describe a pair of Dirac points when they come close together in the vicinity of a time-reversal-invariant momentum (TRIM), two merging scenarios are possible corresponding to the following two universal Hamiltonians [32,33]:

$$
\begin{gathered}
\mathcal{H}_{+-}=\left(\Delta+\frac{q_{x}^{2}}{2 m}\right) \sigma_{\mu}+c q_{y} \sigma_{\nu}, \\
\mathcal{H}_{++}=\left(\Delta+\frac{q_{x}^{2}-q_{y}^{2}}{2 m}\right) \sigma_{\mu}+\frac{q_{x} q_{y}}{m^{\prime}} \sigma_{\nu},
\end{gathered}
$$

where $\sigma_{\mu}, \sigma_{v}$ are two Pauli matrices and $\Delta, c, m$, and $m^{\prime}$ are parameters. Actually, because of time-reversal symmetry, there is not much choice in the Pauli matrices: in the $(+-)$ case $\sigma_{v}$ should be $\sigma_{y}$ and in the $(++)$ case, the $\sigma_{y}$ matrix should not appear in the Hamiltonian.

Making an expansion around each Dirac point, the first (respectively second) Hamiltonian describes two Dirac points with opposite (respectively equal) winding numbers located at position $\left(q_{x}, q_{y}\right)=(\xi \sqrt{-2 m \Delta}, 0)$ if $\Delta<0$. If $\Delta>0$, Dirac points only exist in the second case and are located at $\left(q_{x}, q_{y}\right)=(0, \xi \sqrt{2 m \Delta})$. Here, the winding number \pm 1 associated with a Dirac point is actually defined with respect to the winding plane $\left(\sigma_{\mu}, \sigma_{v}\right)$, which can generally be parameter dependent. This is indeed the case in situations encountered in Ref. [25] and in this paper: when varying a parameter of the Hamiltonian (here the parameter $t \in[0,1]$ ), the orientation of the winding plane (defined by a great circle on the Bloch sphere) varies continuously. We shall see here that a pair of Dirac points emerges following the $\mathcal{H}_{+-}$scenario with $\left(\sigma_{\mu}, \sigma_{v}\right)=\left(\sigma_{z}, \sigma_{y}\right)$, and merges following the $\mathcal{H}_{++}$scenario with $\left(\sigma_{\mu}, \sigma_{v}\right)=\left(\sigma_{z}, \sigma_{x}\right)$. In order to describe the continuous evolution of the winding planes attached to each Dirac point, we recently introduced the notion of a winding vector [25]

$$
\vec{w}=\frac{1}{2 \pi} \int \vec{n} \times d \vec{n}
$$

with the normalized pseudospin magnetic field $\vec{n}(\mathbf{k})=\vec{h} /|\vec{h}|$. $\vec{w}$ is the unit vector normal to the oriented winding plane of $\vec{n}$. The winding vector is a parameter-dependent vectorial quantity, supplemental to the more familiar winding number in describing a band crossing. This notion becomes essential when describing a pair of Dirac points in close vicinity since the relative orientation of the winding plane can have physical consequences. For a Hamiltonian of the form

$$
\mathcal{H}=v_{x} q_{x} \sigma_{\mu}+v_{y} q_{y} \sigma_{\nu},
$$

with $\sigma_{\mu}=\vec{u}_{\mu} \cdot \vec{\sigma}$ and $\sigma_{v}=\vec{u}_{v} \cdot \vec{\sigma}$, we simply have

$$
\vec{w}=\operatorname{sgn}\left(v_{x} v_{y}\right) \vec{u}_{\mu} \times \vec{u}_{\nu} .
$$

In the above equation, it is implicitly assumed that $\left\{\vec{u}_{x}, \vec{u}_{y}, \vec{u}_{z}\right\}$ is an oriented basis.
Finally, we note that a $\sigma_{0}$ term with momentum dependence is important for the flatness of bands in the energy spectrum but not important for wave functions and the topological characterization of a band contact point.

\section{Reduction from three- to two-band effective Hamiltonians}

In order to characterize the distortion of the flat band and the evolution of Dirac points in the three-band system, we introduce local $2 \times 2$ effective Hamiltonians. In order to do this, we proceed in three steps. (1) Because the contact points move along the antidiagonal (lower bands) or the diagonal (upper bands), we will first diagonalize $H(\mathbf{k})$ along these two directions for arbitrary $t$. A great help in this process comes from the mirror symmetries. In this way, we will obtain useful eigenvector bases (see Appendix A). (2) Next, for a specific $\mathbf{k}_{0}$ point along these directions, we will rewrite the Bloch Hamiltonian in the eigenvector basis at $\mathbf{k}_{0}$ and then expand in $\mathbf{q}=\mathbf{k}-\mathbf{k}_{0}$ up to first or second order. In the following, $\mathbf{k}_{0}$ will be chosen either as a TRIM ( $\Gamma$ or $M$ points) or one of the Dirac points $\mathbf{k}_{D}$. (3) The third step consists in projecting from three to two bands to obtain an effective two-band Hamiltonian for Dirac points.

In the following, we study the lower (Sec. III) and upper Dirac points (Sec. IV) in turn.

\section{LOWER DIRAC POINTS}

The lower Dirac points move along the antidiagonal direction $k_{y}=-k_{x}$ from $M$ to $\Gamma$ point as $t$ is varied from 0 to 1 (see Sec. II B and Fig. 3). Specifically, their positions and energy are given by

$$
\xi \mathbf{k}_{a}=\xi\left(2 \phi_{a},-2 \phi_{a}\right) \text { and } E_{a}=-2 \cos \phi_{a},
$$

where $\xi= \pm 1$ is a valley index and the angle $\phi_{a} \geqslant 0$ is defined by

$$
\cos \phi_{a}=t
$$

\section{A. Basis along the antidiagonal}

The Bloch Hamiltonian commutes with the mirror symmetry operator $S_{a}(\mathbf{k})$ everywhere along the antidiagonal line $\left(k_{y}=-k_{x}\right)$ in the Brillouin zone. As detailed in Appendix A, this facilitates the diagonalization of the Hamiltonian using common eigenstates with the symmetry operator, which we denote as $\left(\left|u_{+}\right\rangle,\left|u_{-}\right\rangle,\left|u_{3}\right\rangle\right)$. We then form the unitary matrix $L\left(k_{x}\right)=\left(\left|u_{+}\right\rangle\left|u_{-}\right\rangle\left|u_{3}\right\rangle\right)$ that allows us to write the Bloch Hamiltonian in its eigenbasis as

$$
\tilde{H}_{a}\left(k_{x},-k_{x}\right)=\operatorname{diag}\left(E_{+}, E_{-}, E_{3}\right)
$$

with $\quad E_{ \pm}\left(k_{x}\right)=t \pm \Delta_{a}, \quad E_{3}=E_{a}=-2 t, \quad$ where $\Delta_{a}=$ $\sqrt{t^{2}+8 \cos ^{2} \frac{k_{x}}{2}}$. The largest eigenvalue is $E_{+}$and the second is $E_{-} \geqslant E_{3}$ if $t \geqslant \cos \left(k_{x} / 2\right)$ and $E_{3} \geqslant E_{-}$otherwise. Along the antidiagonal line, they describe the three energy bands. At the Dirac point $k_{x}=2 \phi_{a}$, the lower two bands become degenerate $E_{3}=E_{-}=-2 t$ and separated from the third band $E_{+}=4 t$. We emphasize that, while there is always a gauge freedom in the choice of the set of eigenvectors, we use here an eigenbasis which connects smoothly the two limiting cases from $t=0$ to 1 . 
By writing the original Hamiltonian (1) in the new basis $\tilde{H}(\mathbf{k})=L^{\dagger}\left(k_{x}\right) H(\mathbf{k}) L\left(k_{x}\right)$, we now want to successively describe the vicinity of the $M$ point, the $\Gamma$ point, and the Dirac points (and not limited to $k_{y}=-k_{x}$ ), by appropriate expansions.

\section{B. Close to the Lieb limit: Emergence of Dirac points at $M$ point}

This is the limit $t \rightarrow 0$ when the lower Dirac points pair emerge from the triple crossing at $M=(\pi, \pi)$ point of the Lieb lattice along the antidiagonal line. Upon a momentum expansion at $M$ point for small $\mathbf{q}$, we get $\tilde{H}_{M}(\mathbf{q})=$ $L(\pi)^{\dagger} H(M+\mathbf{q}) L(\pi)$ in the form

$$
\tilde{H}_{M}(\mathbf{q})=\left(\begin{array}{c|cc}
2 t & i q_{\perp} & i \sqrt{2} t q_{\|} \\
\hline \ldots & 0 & -i q_{\|}+\frac{q_{\perp} q_{\|}}{\sqrt{2}} \\
\ldots & \ldots & -2 t
\end{array}\right)
$$

with $q_{\|} \equiv \frac{q_{x}+q_{y}}{\sqrt{2}}$ ("diagonal" direction) and $q_{\perp} \equiv \frac{q_{y}-q_{x}}{\sqrt{2}}$ ("antidiagonal" direction). We keep the second order in $q_{\|}, q_{\perp}$, and $t$ in the relevant blocks (indeed, we will see that the Dirac points correspond to $\left|q_{\perp}\right| \sim t$ ). The vertical/horizontal lines in the matrix merely serve as an eye guide separating the subspaces $\left(\left|u_{+}\right\rangle\right)$and $\left(\left|u_{-}\right\rangle,\left|u_{3}\right\rangle\right)$ which the matrix operates.

We then eliminate the highest band using second-order perturbation theory. Following the Löwdin method (see Appendix B) [34] with typical energy $E_{0}=E_{a}=-2 t$, we obtain an effective $2 \times 2$ Hamiltonian acting in $\left(\left|u_{-}\right\rangle,\left|u_{3}\right\rangle\right)$ subspace as

$$
\mathcal{H}_{M}(\mathbf{q}) \simeq-\left(t+\frac{q_{\perp}^{2}}{8 t}\right) \sigma_{0}+q_{\|} \sigma_{y}+\left(t-\frac{q_{\perp}^{2}}{8 t}\right) \sigma_{z}
$$

at first order in $t$. Apart from the identity term $\sigma_{0}$, it has the form of the universal Hamiltonian $\mathcal{H}_{+-}$[see Eq. (3)], with $\sigma_{\mu}=\sigma_{z}$ and $\sigma_{v}=\sigma_{y}$. It describes the merging of two Dirac points located at $\left(q_{\|}, q_{\perp}\right)=(0, \xi 2 \sqrt{2} t)$ and of opposite winding numbers in the $\sigma_{y}-\sigma_{z}$ plane. The winding vectors are $\vec{w}_{\xi}=-\xi \vec{u}_{x}$.

At second order in $t$, the effective two-band Hamiltonian (12) gets an additional contribution, which, as we show below, is responsible of a rotation of the winding vectors:

$$
\frac{q_{\|} q_{\perp}}{2 \sqrt{2}} \sigma_{x} .
$$

Upon the substitution $q_{\perp} \rightarrow \xi 2 \sqrt{2} t+q_{\perp}$, the linearized Hamiltonian near the Dirac points becomes

$$
\mathcal{H}_{a}(\mathbf{q}) \simeq\left(-2 t-\xi \frac{q_{\perp}}{\sqrt{2}}\right) \sigma_{0}+q_{\|}\left(\sigma_{y}+\xi t \sigma_{x}\right)-\xi \frac{q_{\perp}}{\sqrt{2}} \sigma_{z} .
$$

The Dirac cones are critically tilted because of the fine tuning of the $q_{\perp} \sigma_{0}$ term with respect to the $q_{\perp} \sigma_{z}$ term. Also, there is some mixing between $\sigma_{y}$ and $\sigma_{x}$ indicating that the winding vectors rotate. Indeed $\vec{w}_{\xi}=-\xi \vec{u}_{x}+t \vec{u}_{y}$, which evolve from $-\xi \vec{u}_{x}$ (i.e., antiparallel) at small $t$ to $\vec{u}_{y}$ (i.e., parallel) when increasing $t$.

We remark that the effective Hamiltonian near the $M$ point has a peculiar structure. It results from an expansion a priori valid for small q. But near the merging when $t \rightarrow 0$, one cannot restrict to the two lowest bands since three bands become degenerate in the Lieb limit. Moreover, in this limit, the curvature of the spectrum goes to infinity, which is natural since the spectrum at the merging is indeed conical. The purpose here is to show that the Dirac points show opposite winding numbers and emerge following the $(+-)$ scenario. It should not be applied strictly at the Lieb limit $t=0$ (see the corresponding discussion in Appendix C).

\section{Close to the kagome limit: Merging of Dirac points at $\Gamma$ point}

Close to the kagome limit $(t=1)$, we consider an expansion of the Hamiltonian at the $\Gamma=(0,0)$ point to give $\tilde{H}_{\Gamma}(\mathbf{q})=L(0)^{\dagger} H(\Gamma+\mathbf{q}) L(0)$ as

$$
\tilde{H}_{\Gamma}(\mathbf{q})=\left(\begin{array}{c|cc}
4 & -i q_{\perp} & i \sqrt{3} q_{\|} \\
\hline \ldots & -2-\frac{2 \epsilon}{3}+\frac{q_{\perp}^{2}}{3} & -\frac{q_{\|} q_{\perp}}{\sqrt{3}} \\
\ldots & \ldots & -2+2 \epsilon+q_{\|}^{2}
\end{array}\right)
$$

with $t=1-\epsilon$ and $\epsilon \rightarrow 0$. Similarly to the previous section we keep up to the second order in the variables $q_{\|}, q_{\perp}$ and first order in $\epsilon$ (as we will see that the Dirac points correspond to $\left.q_{\perp}^{2} \sim \epsilon\right)$ in the relevant matrix elements. Because we will treat the highest band as a perturbation to the lower subspace, we only need to consider the off-diagonal blocks at first order and the highest band energy at zeroth order. From the Löwdin method (see Appendix B), we arrive at the effective two-band Hamiltonian

$$
\begin{aligned}
\mathcal{H}_{\Gamma}(\mathbf{q})= & \left(-2+\frac{2 \epsilon}{3}+\frac{q_{\|}^{2}}{4}+\frac{q_{\perp}^{2}}{12}\right) \sigma_{0} \\
& +\left(\frac{q_{\perp}^{2}}{12}-\frac{q_{\|}^{2}}{4}-\frac{4 \epsilon}{3}\right) \sigma_{z}-\frac{q_{\|} q_{\perp}}{2 \sqrt{3}} \sigma_{x}
\end{aligned}
$$

at second order in $q$ and first order in $\epsilon$. Aside from the identity term, it has the form of the universal Hamiltonian $\mathcal{H}_{++}$ [see Eq. (4)] with $\sigma_{\mu}=\sigma_{z}$ and $\sigma_{v}=\sigma_{x}$, following the ( ++ ) merging scenario. It describes the merging of two Dirac points located at $\left(q_{\|}, q_{\perp}\right)=(0,-\xi 4 \sqrt{\epsilon})$ if $\epsilon>0$ [beware that the $+\mathbf{k}_{a}$ Dirac point is at $\left.q_{\perp}=-4 \sqrt{\epsilon}\right]$ and of identical winding numbers in the $\sigma_{x}-\sigma_{z}$ plane. Note that for $\epsilon<0$, the merging is along the $q_{\|}$direction as the Dirac points are located at $\left(q_{\|}, q_{\perp}\right)=(\xi 4 \sqrt{-\epsilon / 3}, 0)$. This agrees with the model of Eq. (4) showing the emergence for $t \leqslant 1$ as well as $t \geqslant 1$.

When $\epsilon=0$, this Hamiltonian describes a quadratic band crossing in the kagome limit. A similar Hamiltonian was obtained by Ref. [16] in the triangular version of the kagome lattice. However, their Hamiltonian [see Eq. (2.6) in Ref. [16] with $d_{3}=0$ ] breaks time-reversal symmetry which is not possible at the $\Gamma$ point. This is due to taking the Pauli matrices $\sigma_{x}, \sigma_{y}$ instead of $\sigma_{z}, \sigma_{x}$ in the Hamiltonian $\mathcal{H}_{++}$[see Eq. (4)].

Finding a rotation of the winding vector requires performing a computation at third order in $q \sim \sqrt{\epsilon}$ in Eq. (15). The Löwdin method then gives the additional terms as

$$
\left(\frac{q_{\perp}^{2}-q_{\|}^{2}}{4 \sqrt{6}}-\frac{4 \epsilon}{3 \sqrt{6}}\right) q_{\|} \sigma_{y} .
$$


Upon the substitution $q_{\perp} \rightarrow-\xi 4 \sqrt{\epsilon}+q_{\perp}$, the linearized Hamiltonian near the Dirac points becomes

$$
\begin{aligned}
\mathcal{H}_{a}(\mathbf{q}) \simeq & \left(-2 t-\xi \frac{2}{3} \sqrt{\epsilon} q_{\perp}\right) \sigma_{0}-\xi \frac{2}{3} \sqrt{\epsilon} q_{\perp} \sigma_{z} \\
& +\xi 2 \sqrt{\frac{\epsilon}{3}} q_{\|}\left(\sigma_{x}+\xi \frac{2}{3} \sqrt{2 \epsilon} \sigma_{y}\right) .
\end{aligned}
$$

The winding vector is $\vec{w}_{\xi}=\vec{u}_{y}-\xi \frac{2}{3} \sqrt{2 \epsilon} \vec{u}_{x}$, when $\epsilon$ is small.

\section{Motion of the lower Dirac points}

We now find the paradoxical situation where a $(+-)$ pair of Dirac points near $M$ (when $t \simeq 0$ ) continuously evolves into a $(++)$ pair near $\Gamma$ (when $t \simeq 1$ ). Naively, one expects that a topological charge conservation, which holds locally in the momentum space, should also hold globally in the full BZ. Here, the global charge seems to change from 0 (for $t=0$ ) to +2 (for $t=1$ ).

To solve the apparent paradox, we now follow the evolution of the Dirac points and determine the evolution of the associated winding vector. We make an expansion following the Dirac point at $\mathbf{k}_{a}=\left(2 \phi_{a},-2 \phi_{a}\right)$, where the angle $\phi_{a}$ is defined in Eq. (9), to get $\tilde{H}_{a}(\mathbf{q})=L\left(2 \phi_{a}\right)^{\dagger} H\left(\mathbf{k}_{a}+\mathbf{q}\right) L\left(2 \phi_{a}\right)$ as

$\tilde{H}_{a}(\mathbf{q})=\left(\begin{array}{c|cc}4 t & \mathcal{O}(\mathbf{q}) & \mathcal{O}(\mathbf{q}) \\ \hline \ldots & E_{a}-\frac{2 \sqrt{2}}{3} \sin \phi_{a} q_{\perp} & \sqrt{\frac{2}{3}} \sin \phi_{a} e^{-i \phi_{a}} q_{\|} \\ \ldots & \ldots & E_{a}\end{array}\right)$,

where $E_{a}=-2 \cos \phi_{a}=-2 t$. For the other Dirac point at $-\mathbf{k}_{a}$, one should replace $\phi_{a}$ by $-\phi_{a}$. Because we are now interested in a Dirac Hamiltonian, which is linear in momentum, and in contrast to the two cases previously studied, we only need to keep the momentum variable up to the linear order. Corrections from the third band are necessarily of the second order and can be neglected. The effective Hamiltonian can therefore be read off from the $2 \times 2$ block spanned by $\left(\left|u_{-}\right\rangle,\left|u_{3}\right\rangle\right)$ (see Appendix A) as

$$
\mathcal{H}_{a}(\mathbf{q}) \simeq\left(E_{a}-v_{\perp}^{0} q_{\perp}\right) \sigma_{0}+v_{\|} q_{\|} \sigma_{\phi_{a}}-v_{\perp} q_{\perp} \sigma_{z},
$$

with the (positive) velocities

$$
v_{\|}=\sqrt{\frac{2}{3}} \sin \phi_{a}, \quad v_{\perp}=v_{\perp}^{0}=\frac{v_{\|}}{\sqrt{3}} .
$$

In the $t \rightarrow 0$ limit, $v_{\|} \simeq \sqrt{2 / 3}$ and $v_{\perp}=v_{\perp}^{0} \simeq \sqrt{2} / 3$; whereas when $t=1-\epsilon \rightarrow 1, v_{\|} \simeq 2 \sqrt{\epsilon / 3}$ and $v_{\perp}=v_{\perp}^{0} \simeq$ $2 \sqrt{\epsilon} / 3$. These velocities agree with that found in the expansion near the $M$ point (when $t \rightarrow 1$ ) but there is a numerical discrepancy with that found near the $\Gamma$ point (when $t \rightarrow 0$ ).

The Pauli matrix $\sigma_{\phi_{a}} \equiv \cos \phi_{a} \sigma_{x}+\sin \phi_{a} \sigma_{y}$, together with $\sigma_{z}$, defines the pseudospin winding plane. This plane evolves in the pseudospin space from $\left(\sigma_{y}, \sigma_{z}\right)$ to $\left(\sigma_{x}, \sigma_{z}\right)$ with $\phi_{a}$ going from $\pi / 2(t=0)$ to $0(t=1)$ (see Fig. 6). For the two Dirac points at $\xi \mathbf{k}_{a}=\xi\left(2 \phi_{a},-2 \phi_{a}\right)$, following Eq. (7), the associated winding vectors are

$$
\begin{aligned}
\vec{w}_{\xi} & =-\xi \sin \phi_{a} \vec{u}_{x}+\cos \phi_{a} \vec{u}_{y} \\
& =-\xi \sqrt{1-t^{2}} \vec{u}_{x}+t \vec{u}_{y} .
\end{aligned}
$$

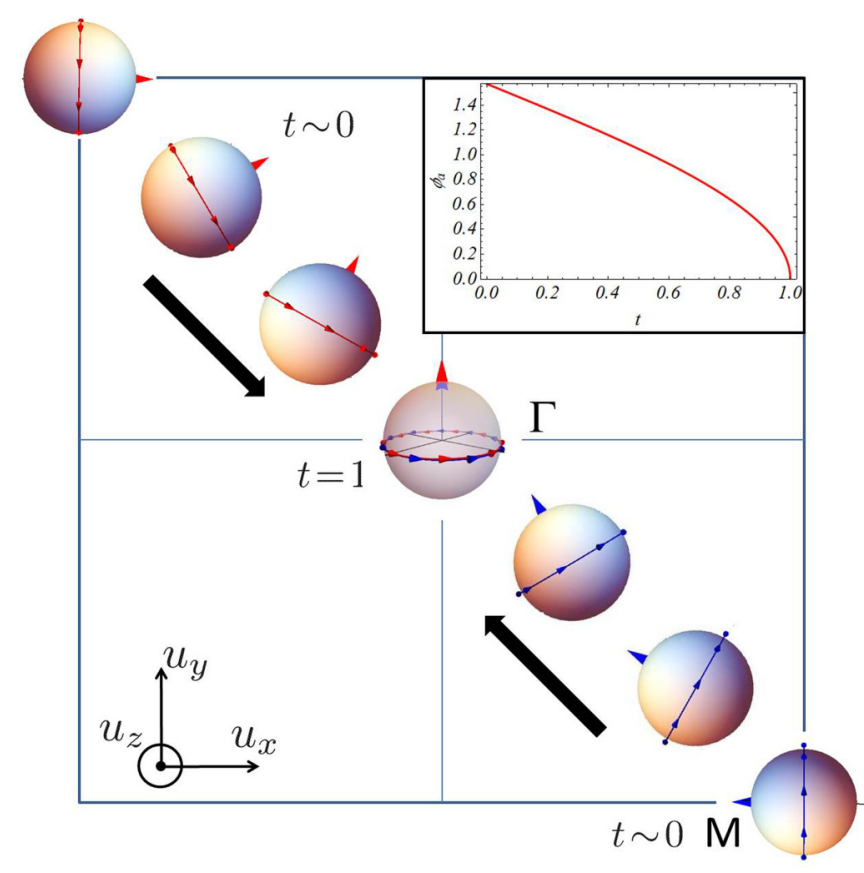

FIG. 6. Winding vector evolution as a function of $t$ for the lower Dirac points. The inset shows the angle $\phi_{a}$ as a function of $t$. The position of Dirac points is $\xi \mathbf{k}_{a}=\xi\left(2 \phi_{a},-2 \phi_{a}\right)$.

In the $t \rightarrow 0$ limit, $\vec{w}_{\xi} \simeq-\xi \vec{u}_{x}+t \vec{u}_{y}$; whereas when $t=1-$ $\epsilon \rightarrow 1, \vec{w}_{\xi} \simeq-\xi \sqrt{2 \epsilon} \vec{u}_{x}+\vec{u}_{y}$. Apart from a factor of $\frac{2}{3}$ when $t$ is close to 1 , this agrees with the results of the expansions near the $M$ and $\Gamma$ points.

As an aside, we note that the $\sigma_{0}$ term modifies the dispersion of the Dirac cone quite drastically. As $v_{\perp}=v_{\perp}^{0}$ for all $t$, the cones are critically tilted [also known as type III Dirac-Weyl cones (see, e.g., Ref. [8] and references therein)]: the velocities of excitations along the $\perp$ direction are $v_{\min }=$ $v_{\perp}-v_{\perp}^{0}=0$ and $v_{\max }=v_{\perp}+v_{\perp}^{0}=2 v_{\perp}$. The pair of Dirac points is connected by a flat energy line (see red dots in Figs. 4 and 5).

In summary, for the crossings in the lower two bands, Eqs. (12) and (16) describe the emergence/merging of two Dirac cones with different winding scenarios. In the single Dirac cone Hamiltonian (20), we follow the evolving winding vector and show how the apparent winding number gets reversed as $t$ is varied.

\section{UPPER DIRAC POINTS}

For the upper Dirac point pair, we perform an analysis similar to the one contained in the last section. The Dirac points move along the diagonal line $k_{y}=k_{x}$, with their positions and energy (Fig. 3) given by

$$
\xi \mathbf{k}_{d}=\xi\left(2 \phi_{d}, 2 \phi_{d}\right) \text { and } E_{d}=2 \cos \phi_{d}
$$

and specified by the angle $\phi_{d} \geqslant 0$ such that

$$
\cos \phi_{d}=\frac{\sqrt{1+8 t^{2}}-1}{4 t} .
$$

The upper Dirac points that emerge from the $M$ point $(t=0)$ move to a fixed position $\xi \mathbf{K}=\xi(2 \pi / 3,2 \pi / 3)$ in the kagome 
limit $(t=1)$ and do not merge (in contrast to the lower Dirac points pair).

\section{A. Basis along the diagonal}

The Bloch Hamiltonian commutes with the mirror symmetry operator $S_{d}(\mathbf{k})$ everywhere along the diagonal line $\left(k_{y}=\right.$ $k_{x}$ ) in the Brillouin zone. As detailed in Appendix A, a common basis of eigenvectors of the Hamiltonian and the symmetry operator is found, which we denote as $\left(\left|v_{1}\right\rangle,\left|v_{+}\right\rangle,\left|v_{-}\right\rangle\right)$. We emphasize that this basis furnishes a smooth interpolation between the two limits $t=0$ and 1 . The unitary matrix is defined by $U\left(k_{x}\right) \equiv\left(\left|v_{1}\right\rangle\left|v_{+}\right\rangle\left|v_{-}\right\rangle\right)$and written in this basis, the Hamiltonian along the diagonal line is given by

$$
\tilde{H}_{d}\left(k_{x}, k_{x}\right)=\operatorname{diag}\left(E_{1}, E_{+}, E_{-}\right)
$$

with $E_{1}=-2 t \cos k_{x}$ and $E_{ \pm}=t \cos k_{x} \pm \Delta_{d}$, where $\Delta_{d}=\sqrt{t^{2} \cos ^{2} k_{x}+8 \cos ^{2} \frac{k_{x}}{2}}$. We see that the two upper bands become degenerate at energy $E_{1}=E_{+}=$ $2 \cos \phi_{d}=-2 t \cos \left(2 \phi_{d}\right)$ separated from the lowest band $E_{-}=-4 \cos \phi_{d}$ at the Dirac point.

\section{B. Close to the Lieb limit: Emergence of Dirac points at $M$ point}

At $M=(\pi, \pi)$, the unitary matrix $U(\pi)=\left(\left|v_{1}\right\rangle\left|v_{2}\right\rangle\left|v_{3}\right\rangle\right)$ is equal to $L(\pi)=\left(\left|u_{1}\right\rangle\left|u_{2}\right\rangle\left|u_{3}\right\rangle\right)$ as $\left|v_{j}\right\rangle=\left|u_{j}\right\rangle$. Instead of Eq. (11), we focus on the upper two bands to obtain $\tilde{H}_{M}(\mathbf{q})=$ $U(\pi)^{\dagger} H(M+\mathbf{q}) U(\pi)$ as

$$
\tilde{H}_{M}(\mathbf{q})=\left(\begin{array}{cc|c}
2 t & i q_{\perp}+\frac{q_{\|}^{2}+q_{\perp}^{2}}{2 \sqrt{2}} & i \sqrt{2} t q_{\|} \\
\ldots & 0 & -i q_{\|} \\
\hline \cdots & \cdots & -2 t
\end{array}\right)
$$

at second order in the variables $q_{\|}, q_{\perp}$, and $t$ (as we will see that the Dirac points correspond to $q_{\|}^{2} \sim t^{2}$ ) in the relevant blocks. Using the Löwdin method with typical energy $E_{0}=$ $E_{d} \simeq 2 t$, when $t \rightarrow 0$ (see Appendix B), we arrive at the effective two-band Hamiltonian (first order in $t$ )

$$
\mathcal{H}_{M}(\mathbf{q}) \simeq\left(t+\frac{q_{\|}^{2}}{8 t}\right) \sigma_{0}+\left(t-\frac{q_{\|}^{2}}{8 t}\right) \sigma_{z}-q_{\perp} \sigma_{y}
$$

in the subspace of $\left(\left|v_{1}\right\rangle,\left|v_{+}\right\rangle\right)$. Apart from the identity $\sigma_{0}$ term, it takes the form of the universal Hamiltonian $\mathcal{H}_{+-}$[see Eq. (3)] with $\sigma_{\mu}=\sigma_{z}$ and $\sigma_{v}=\sigma_{y}$, describing the emergence of two Dirac points at $\left(q_{\|}, q_{\perp}\right)=(-\xi 2 \sqrt{2} t, 0)$ (beware that the $+\mathbf{k}_{d}$ Dirac point is at $q_{\|}=-2 \sqrt{2} t$ ) of opposite winding vectors $\vec{w}_{\xi}=\xi \vec{u}_{x}$ for $t \simeq 0$. The Hamiltonians (12) and (27) are similar upon exchanging the diagonal $q_{\|}$and the antidiagonal $q_{\perp}$. They both describe a pair of critically tilted Dirac cones. The critical tilt is true for the lower cones but actually not for the upper ones, as seen on Fig. 4(c), and because of neglected higher-order terms [cf. $v_{\|}^{0} \neq v_{\|}$in Eq. (31)].

At second order in $t$, the effective two-band Hamiltonian (27) gets an additional contribution:

$$
\frac{q_{\perp}^{2}}{2 \sqrt{2}} \sigma_{x}
$$

This term is similar to Eq. (13) except that $q_{\|} q_{\perp}$ is replaced by $q_{\perp}^{2}$. Whereas Eq. (13) leads to a rotation of the winding vector, it is not the case of Eq. (28). Indeed, upon the substitution $q_{\|} \rightarrow-\xi 2 \sqrt{2} t+q_{\|}$, the linearized Hamiltonian near the Dirac points reads as

$$
\mathcal{H}_{d} \simeq\left(2 t-\frac{\xi}{\sqrt{2}} q_{\|}\right) \sigma_{0}-q_{\perp} \sigma_{y}+\frac{\xi}{\sqrt{2}} q_{\|} \sigma_{z} .
$$

In contrast to the lower Dirac points pair, here there is no rotation of the winding vector, which is $\vec{w}_{\xi}=\xi \vec{u}_{x}$ independently of $t$. Since the Dirac cones do not approach each other again in the kagome limit $t \rightarrow 1$, this concludes the analysis for double Dirac cones in the upper two bands.

\section{Motion of the upper Dirac points}

To follow the evolution of a single Dirac cone, we first expand the Hamiltonian $\tilde{H}_{d}(\mathbf{q})=U\left(\xi 2 \phi_{d}\right)^{\dagger} H\left(\mathbf{k}_{d}+\mathbf{q}\right) U\left(\xi 2 \phi_{d}\right)$ at the position $\xi \mathbf{k}_{d}$ keeping linear order in $\mathbf{q}$. The effective Hamiltonian for the Dirac point is then obtained directly from the block Hamiltonian in the $\left(\left|v_{1}\right\rangle,\left|v_{+}\right\rangle\right)$subspace (see Appendix A) as

$$
\mathcal{H}_{d}(\mathbf{q})=\left(E_{d}-\xi v_{\|}^{0} q_{\|}\right) \sigma_{0}+\xi v_{\|} q_{\|} \sigma_{z}-v_{\perp} q_{\perp} \sigma_{y},
$$

where $E_{d}=2 \cos \phi_{d}=\frac{\sqrt{1+8 t^{2}}-1}{2 t}$ and we defined the (positive) velocities

$$
\begin{aligned}
v_{\|}^{0} & =\frac{\sqrt{2} \sin \left(3 \phi_{d}\right)}{3 \cos \left(2 \phi_{d}\right)}, \quad v_{\|}=\frac{\sin \phi_{d}+\sin \left(3 \phi_{d}\right) / 3}{-\sqrt{2} \cos \left(2 \phi_{d}\right)}, \\
\text { and } v_{\perp} & =\sqrt{\frac{2}{3}} \sin \phi_{d} .
\end{aligned}
$$

In the $t \rightarrow 0$ limit, $v_{\|}^{0} \simeq v_{\|} \simeq \sqrt{2} / 3$ (critical tilt) and $v_{\perp} \simeq$ $\sqrt{2 / 3}$; whereas when $t \rightarrow 1, v_{\|}^{0} \simeq 0$ (no tilt), $v_{\|} \simeq \sqrt{3 / 2}$, and $v_{\perp} \simeq 1 / \sqrt{2}$. Except when $t=0, v_{\|}^{0} \neq v_{\|}$, which means that the tilt is not critical, an effect not captured by Eq. (27).

When $t$ varies from 0 to 1 , the upper pair emerges from the $M$ point and reaches two fixed points $\xi \mathbf{K}=\xi\left(\frac{2 \pi}{3}, \frac{2 \pi}{3}\right)$ [see Fig. 3(a)]. Following Eq. (7), the winding vectors associated to the two Dirac points $\xi \mathbf{k}_{d}$ are $\vec{w}_{\xi}=\xi \vec{u}_{x}$. In this case, there is no rotation of the winding vectors, which remain antiparallel.

\section{DISCUSSION AND CONCLUSION}

We have studied in detail the evolution of Dirac points in a tight-binding model interpolating between two well-known three-band models, the Lieb and the kagome lattices. In both limits, the energy spectrum exhibits a flat band, symmetrically positioned in the middle of a conical spectrum for the Lieb lattice, at a quadratic touching with a dispersive band for the kagome lattice. One of the interests of this study is to interpolate between two different flat bands: in the Lieb case, the flat band is topological (as a result of the lattice being bipartite), whereas in the kagome case it results from destructive interferences. In the first case, the flat band resists the introduction of a perpendicular magnetic field, whereas it is destroyed in the second case (see the corresponding Hofstadter butterflies in Ref. [14]).

Starting from the kagome lattice, the quadratic contact point is split into two Dirac points characterized by the same winding number, following a well-characterized 
universal scenario. Upon further variation of the interpolating parameter, these two Dirac points merge, following another well-characterized merging scenario for a pair of Dirac points with opposite winding numbers. To solve this apparent contradiction and to characterize the topological properties of these contact points, we have reduced the full $3 \times 3$ Hamiltonian to effective $2 \times 2$ Hamiltonians using two different approaches (see below). During the evolution between the two limits, the effective Hamiltonian (or more precisely its effective pseudomagnetic field $\vec{h}$ ) rotates in pseudospin space, leading to the notion of winding vector. The winding vectors of a pair evolve from a parallel to an antiparallel alignment.

This work poses questions about a systematic way to obtain effective two-band Hamiltonians describing contact points in multiband models. Here, we have essentially used two different strategies: (i) an expansion of the three-band Bloch Hamiltonian at second (or third) order in the wave vector in the vicinity of a TRIM, followed by a projection (using second-order perturbation theory) in order to capture the pair of contact points located away from the TRIM; (ii) an expansion of the three-band Bloch Hamiltonian at first order in the wave vector directly in the vicinity of the contact point at $\mathbf{k}_{D}$ followed by a naive projection (without the need of perturbation theory).

Such a reduction from a multiband to an effective $2 \times 2$ Hamiltonian for a pair of Dirac points is intrinsically local in $\mathbf{k}$ space because it is local in energy space: it can not be done in the whole Brillouin zone. It is important to realize that such a procedure carries some arbitrariness as it depends on a choice of eigenbasis. This is especially crucial for procedure (ii), which depends on an eigenbasis at the Dirac point. Indeed, any linear combination of degenerate eigenvectors produces a valid alternative eigenbasis. The consequence is that the direction of the winding vector for a single Dirac point has no absolute meaning and can be changed almost at will by choosing a different basis of degenerate eigenvectors.

However, the relative direction between the winding vectors of the two Dirac points within a pair has an absolute meaning. This appears clearly when computing the winding vectors in the same basis for the two Dirac points, as done in procedure (i). In other words, the effective $2 \times 2$ Hamiltonian should describe both Dirac points at once. This is typically a "universal" Hamiltonian, similar to $\mathcal{H}_{+-}$or $\mathcal{H}_{++}$, built from a TRIM (either $\Gamma$ or $M$ point in the present context). As we have shown, higher-order terms in momentum provide corrections to these universal Hamiltonians that can describe the rotation of the winding vectors.

In the future, it would be interesting to fully characterize a multiband problem by introducing angles that describe band couplings between more than two bands and that are valid in the complete Brillouin zone and not only locally in reciprocal space. Here, we have seen how the Lieb point splits in four Dirac points as the hopping $t$ becomes finite. It would be interesting to systematically study the different splitting scenarios of degeneracy points between three bands.

\section{ACKNOWLEDGMENTS}

We thank G. Abramovici for useful discussions on the Lieb-kagome model. L.-K.L. is supported by the Thousand
Young Talents Program of China. We thank the Institute for Advanced Study of the Tsinghua University in Beijing for hosting us while part of this work was being performed.

\section{APPENDIX A: BASIS ALONG THE ANTIDIAGONAL AND THE DIAGONAL}

This Appendix provides details of the derivation of the two distinct eigenbases $\left(\left|u_{ \pm}\left(k_{x}\right)\right\rangle,\left|u_{3}\left(k_{x}\right)\right\rangle\right)$ and $\left(\left|v_{1}\left(k_{x}\right)\right\rangle,\left|v_{ \pm}\left(k_{x}\right)\right\rangle\right)$ that diagonalize the Bloch Hamiltonian $H(\mathbf{k})$, for any value of the parameter $t$, along respectively the antidiagonal $k_{x}=-k_{y}$ and diagonal $k_{x}=k_{y}$. The key ingredients are the mirror symmetries $S_{a}$ and $S_{d}$ along the diagonal and antidiagonal, respectively.

To start with, it is important to remind that the specific form of the Bloch Hamiltonian $H(\mathbf{k})$ written in Eq. (1) is a direct consequence of the implicit choice of the Bloch basis $|\mathbf{k}, \alpha\rangle=\sum_{\mathbf{R}} e^{i \mathbf{k} \cdot \mathbf{R}}|\mathbf{R}, \alpha\rangle(\alpha=A, B, C)$ that depends only on the Bravais lattice vectors $\mathbf{R}$ (choice known as basis I [35]). The main advantage of this Bloch basis is that the Bloch Hamiltonian matrix $H(\mathbf{k})$ representing the Hamiltonian is periodic $H(\mathbf{k}+\mathbf{G})=H(\mathbf{k})$ for any reciprocal lattice vectors G. However, an important drawback of this representation is that the matrix $H(\mathbf{k})$ has lost the information of the full position of the different atoms in the unit cell. In fact, the latter information is now encoded in the matrices representing the different space symmetries of the lattice, highlighting their key role.

\section{Diagonalization of $H(\mathrm{k})$ along the antidiagonal}

The Bloch matrix representation of the mirror symmetry $S_{a}$ along the real-space antidiagonal is given by

$$
S_{a}(\mathbf{k})=\left(\begin{array}{ccc}
0 & 0 & 1 \\
0 & 1 & 0 \\
1 & 0 & 0
\end{array}\right) .
$$

This symmetry translates into the relation

$$
S_{a}(\mathbf{k})^{\dagger} H\left(k_{x}, k_{y}\right) S_{a}(\mathbf{k})=H\left(-k_{y},-k_{x}\right)
$$

for the Bloch Hamiltonian in reciprocal space. In particular, $S_{a}(\mathbf{k})$ commutes with $H(\mathbf{k})$ on the antidiagonal $k_{x}=-k_{y}$. We therefore diagonalize $S_{a}\left(k_{x},-k_{x}\right)$ to get the eigenvectors

$$
\begin{aligned}
& \left|u_{1}\right\rangle=\frac{1}{\sqrt{2}}(1,0,1)^{T}, \\
& \left|u_{2}\right\rangle=(0,1,0)^{T}, \\
& \left|u_{3}\right\rangle=\frac{1}{\sqrt{2}}(-1,0,1)^{T},
\end{aligned}
$$

with respective eigenvalues $(+1,+1,-1)$. Written in this basis, the Hamiltonian transforms into a block-diagonal matrix

$$
\tilde{H}_{a}\left(k_{x}\right)=\left(\begin{array}{cc|c}
2 t & 2 \sqrt{2} \cos \frac{k_{x}}{2} e^{i \frac{k_{x}}{2}} & 0 \\
\ldots & 0 & 0 \\
\hline \cdots & \cdots & -2 t
\end{array}\right)
$$

in the $\left(\left|u_{1}\right\rangle,\left|u_{2}\right\rangle,\left|u_{3}\right\rangle\right)$ basis. The $2 \times 2$ submatrix in the subspace $\left|u_{1}\right\rangle,\left|u_{2}\right\rangle$ can be rewritten

$$
\mathcal{H}_{u_{1}, u_{2}}\left(k_{x}\right)=t \sigma_{0}+\left(\begin{array}{cc}
\Delta_{a} \cos \theta_{a} & \Delta_{a} \sin \theta_{a} e^{i \frac{k_{x}}{2}} \\
\ldots & -\Delta_{a} \cos \theta_{a}
\end{array}\right)
$$


by introducing the parameters $\Delta_{a}\left(k_{x}\right)=\sqrt{t^{2}+8 \cos ^{2} \frac{k_{x}}{2}}$ and the angle $\theta_{a}\left(k_{x}\right)$ with $\cos \theta_{a}=t / \Delta_{a}$ and $\sin \theta_{a}=$ $2 \sqrt{2} \cos \frac{k_{x}}{2} / \Delta_{a}$. This has the standard form of a two-level problem with its parametric solution.

Therefore, the eigenbasis and their eigenvalues which diagonalize the Hamiltonian $\tilde{H}_{a}\left(k_{x}\right)$ everywhere along the antidiagonal are

$$
\begin{aligned}
& \left|u_{+}\left(k_{x}\right)\right\rangle=\cos \frac{\theta_{a}}{2}\left|u_{1}\right\rangle+\sin \frac{\theta_{a}}{2} e^{-i \frac{k_{x}}{2}}\left|u_{2}\right\rangle, \\
& \left|u_{-}\left(k_{x}\right)\right\rangle=-\sin \frac{\theta_{a}}{2} e^{i \frac{k_{x}}{2}}\left|u_{1}\right\rangle+\cos \frac{\theta_{a}}{2}\left|u_{2}\right\rangle,
\end{aligned}
$$

and $\left|u_{3}\right\rangle$, with their corresponding eigenvalues $E_{ \pm}\left(k_{x}\right)=t \pm$ $\Delta_{a}, E_{3}=-2 t$. Finally, the chosen eigenbasis for $H\left(k_{x},-k_{x}\right)$ reads as

$$
\begin{aligned}
& \left|u_{+}\right\rangle=\frac{1}{\sqrt{2}}\left(\cos \frac{\theta_{a}}{2}, e^{-i \frac{k_{x}}{2}} \sqrt{2} \sin \frac{\theta_{a}}{2}, \cos \frac{\theta_{a}}{2}\right)^{T}, \\
& \left|u_{-}\right\rangle=\frac{1}{\sqrt{2}}\left(-e^{i \frac{k_{x}}{2}} \sin \frac{\theta_{a}}{2}, \sqrt{2} \cos \frac{\theta_{a}}{2},-e^{i \frac{k_{x}}{2}} \sin \frac{\theta_{a}}{2}\right)^{T}, \\
& \left|u_{3}\right\rangle=\frac{1}{\sqrt{2}}(-1,0,1)^{T} .
\end{aligned}
$$

\section{Diagonalization of $\boldsymbol{H}(\mathrm{k})$ along the diagonal}

We now proceed very similarly along the diagonal $k_{x}=k_{y}$. The Bloch matrix representation of the mirror symmetry $S_{d}$ along the real-space diagonal is given by

$$
S_{d}(\mathbf{k})=\left(\begin{array}{ccc}
0 & 0 & e^{i k_{x}} \\
0 & 1 & 0 \\
e^{-i k_{y}} & 0 & 0
\end{array}\right)
$$

This symmetry translates into the relation

$$
S_{d}(\mathbf{k})^{\dagger} H\left(k_{x}, k_{y}\right) S_{d}(\mathbf{k})=H\left(k_{y}, k_{x}\right)
$$

for the Bloch Hamiltonian in reciprocal space. In particular, $S_{d}(\mathbf{k})$ commutes with $H(\mathbf{k})$ on the diagonal $k_{x}=k_{y}$. We therefore diagonalize $S_{d}\left(k_{x}, k_{x}\right)$ to get the eigenvectors

$$
\begin{gathered}
\left|v_{1}\right\rangle=e^{i s \frac{\pi}{2}} \frac{1}{\sqrt{2}}\left(-e^{i \frac{k_{x}}{2}}, 0, e^{-i \frac{k_{x}}{2}}\right)^{T}, \\
\left|v_{2}\right\rangle=(0,1,0)^{T}, \\
\left|v_{3}\right\rangle=e^{i s \frac{\pi}{2}} \frac{1}{\sqrt{2}}\left(e^{i \frac{k_{x}}{2}}, 0, e^{-i \frac{k_{x}}{2}}\right)^{T},
\end{gathered}
$$

with respective eigenvalues $(-1,+1,+1)$, where $s$ is defined as $s=\operatorname{sgn} k_{x}$ if $0<\left|k_{x}\right| \leqslant \pi$ and $s=0$ if $k_{x}=0$. The reason for this phase choice is that we want the kets $\left|v_{j}\right\rangle$ to be real at $\Gamma$ and $M$, to be periodic with the BZ, and that $\left|v_{j}\left(-k_{x}\right)\right\rangle=$ $\left(\left|v_{j}\left(k_{x}\right)\right\rangle\right)^{*}$. Written in this basis, the Hamiltonian thus transforms into a block-diagonal matrix:

$$
\tilde{H}_{d}\left(k_{x}\right)=\left(\begin{array}{c|cc}
-2 t \cos k_{x} & 0 & 0 \\
\hline \ldots & 0 & e^{i s \frac{\pi}{2}} 2 \sqrt{2} \cos \frac{k_{x}}{2} \\
\ldots & \ldots & 2 t \cos k_{x}
\end{array}\right)
$$

in the $\left(\left|v_{1}\right\rangle,\left|v_{2}\right\rangle,\left|v_{3}\right\rangle\right)$ basis. Consider the $2 \times 2$ effective submatrix $\mathcal{H}_{v_{2}, v_{3}}\left(k_{x}\right)$ in the subspace $\left(\left|v_{2}\right\rangle,\left|v_{3}\right\rangle\right)$; we obtain an effective Hamiltonian of the same structure as in Eq. (A5) with the substitution $\Delta_{a}\left(k_{x}\right) \rightarrow \Delta_{d}\left(k_{x}\right)=\sqrt{t^{2} \cos ^{2} k_{x}+8 \cos ^{2} \frac{k_{x}}{2}}$ and the angle $\theta_{a}\left(k_{x}\right) \rightarrow \theta_{d}\left(k_{x}\right)$ with $\cos \theta_{d}=-t \cos k_{x} / \Delta_{d} \geqslant$ 0 and $\sin \theta_{d}=2 \sqrt{2} \cos \frac{k_{x}}{2} / \Delta_{d}$, and the coefficient for the identity term $t \rightarrow t \cos k_{x}$. Thus, a basis that diagonalizes the Hamiltonian $\tilde{H}_{d}\left(k_{x}\right)$ everywhere along the diagonal is

$$
\begin{aligned}
& \left|v_{+}\left(k_{x}\right)\right\rangle=\cos \frac{\theta_{d}}{2}\left|v_{2}\right\rangle+\sin \frac{\theta_{d}}{2} e^{-i s \frac{\pi}{2}}\left|v_{3}\right\rangle, \\
& \left|v_{-}\left(k_{x}\right)\right\rangle=-\sin \frac{\theta_{d}}{2}\left|v_{2}\right\rangle+\cos \frac{\theta_{d}}{2} e^{-i s \frac{\pi}{2}}\left|v_{3}\right\rangle,
\end{aligned}
$$

and $\left|v_{1}\right\rangle$, with the eigenvalues $E_{ \pm}=t \cos k_{x} \pm \Delta_{d}$ and $E_{1}=$ $-2 t \cos k_{x}$. Finally, the chosen eigenbasis for $H\left(k_{x}, k_{x}\right)$ reads as

$$
\begin{aligned}
& \left|v_{1}\right\rangle=\frac{e^{i s \frac{\pi}{2}}}{\sqrt{2}}\left(-e^{i \frac{k_{x}}{2}}, 0, e^{-i \frac{k_{x}}{2}}\right)^{T}, \\
& \left|v_{+}\right\rangle=\frac{1}{\sqrt{2}}\left(e^{i \frac{k_{x}}{2}} \sin \frac{\theta_{d}}{2}, \sqrt{2} \cos \frac{\theta_{d}}{2}, e^{-i \frac{k_{x}}{2}} \sin \frac{\theta_{d}}{2}\right)^{T}, \\
& \left|v_{-}\right\rangle=\frac{1}{\sqrt{2}}\left(e^{i \frac{k_{x}}{2}} \cos \frac{\theta_{d}}{2},-\sqrt{2} \sin \frac{\theta_{d}}{2}, e^{-i \frac{k_{x}}{2}} \cos \frac{\theta_{d}}{2}\right)^{T} .
\end{aligned}
$$

\section{APPENDIX B: LÖWDIN PARTITIONING METHOD FOR THE EFFECTIVE $2 \times 2$ HAMILTONIAN}

The effective Hamiltonian in a subspace can be obtained using the method of Löwdin [34], also called partitioning. Let the Hilbert space be described as the direct sum of two subspaces labeled $\alpha$ and $\beta$. The Hamiltonian then takes the following block form:

$$
H=\left(\begin{array}{c|c}
H_{\alpha \alpha} & H_{\alpha \beta} \\
\hline H_{\beta \alpha} & H_{\beta \beta}
\end{array}\right) .
$$

It is then easy to show that the eigenequation $H|\psi\rangle=E|\psi\rangle$ reads as

$$
\left[H_{\alpha \alpha}+H_{\alpha \beta}\left(E-H_{\beta \beta}\right)^{-1} H_{\beta \alpha}\right]\left|\psi_{\alpha}\right\rangle=E\left|\psi_{\alpha}\right\rangle
$$

in the $\alpha$ subspace, where $|\psi\rangle=\left(\left|\psi_{\alpha}\right\rangle,\left|\psi_{\beta}\right\rangle\right)^{T}$. The operator in the left-hand side of the above equation acts as an effective Hamiltonian in the $\alpha$ subspace, except that it depends on the energy $E$, i.e., (B2) is a self-consistent equation in the spirit of the well-known Brillouin-Wigner perturbation theory. Replacing $E$ in the left-hand side by a typical relevant energy $E=E_{0}$ gives an effective Hamiltonian

$$
\mathcal{H}_{\alpha}=H_{\alpha \alpha}+H_{\alpha \beta}\left(E_{0}-H_{\beta \beta}\right)^{-1} H_{\beta \alpha},
$$

so that the approximate eigenequation replacing (B2) reads as $\mathcal{H}_{\alpha}\left|\psi_{\alpha}\right\rangle \simeq E\left|\psi_{\alpha}\right\rangle$. Compared to a naive projection $\mathcal{H}_{\alpha} \simeq H_{\alpha \alpha}$, this effective Hamiltonian includes corrections $H_{\alpha \beta}\left(E_{0}-H_{\beta \beta}\right)^{-1} H_{\beta \alpha}$ akin to second-order perturbation theory.

Specifically, for the $3 \times 3$ Hamiltonian

$$
H=\left(\begin{array}{cc|c}
E_{1} & a & c \\
a^{*} & E_{2} & b \\
\hline c^{*} & b^{*} & E_{3}
\end{array}\right),
$$


we get the effective $2 \times 2$ Hamiltonian after eliminating the third band which reads as

$$
\mathcal{H}_{\alpha}=\left(\begin{array}{cc}
\tilde{E}_{1} & \tilde{a} \\
\tilde{a}^{*} & \tilde{E}_{2}
\end{array}\right)
$$

with

$$
\begin{aligned}
& \tilde{E}_{1}=E_{1}+\frac{|c|^{2}}{E_{0}-E_{3}}, \quad \tilde{E}_{2}=E_{2}+\frac{|b|^{2}}{E_{0}-E_{3}}, \\
& \tilde{a}=a+\frac{c b^{*}}{E_{0}-E_{3}} .
\end{aligned}
$$

With this method there is some arbitrariness in the choice of $E_{0}$. In this paper, we are interested in describing Dirac points as seen from the nearest TRIM (e.g., $\Gamma$ or $M$ point in the BZ): this means that $E_{0}$ will be chosen to be the energy of the Dirac points, called $E_{a}$ and $E_{d}$ in the main text, in order to properly describe the vicinity of the Dirac points and not the vicinity of the TRIM.

\section{APPENDIX C: GENERALIZED LIEB-KAGOME MODEL}

The effective $2 \times 2$ Hamiltonian (12) describes the evolution of the two lower Dirac points along the antidiagonal, close to the Lieb limit (red points on Fig. 4). Similarly, the Hamiltonian (27) describes the evolution of the two upper Dirac points along the diagonal (blue points on Fig. 4). However, when $t \rightarrow 0$, a two-band description is not appropriate since the four points merge together. The Hamiltonians (12) and (27) provide a correct description of the merging of each pair close to the $M$ point, but not too close. They do not correctly describe the $t=0$ limit. This is obvious when considering the divergence of the $q_{\perp}^{2} / 8 t$ and $q_{\|}^{2} / 8 t$ terms, respectively, in Eqs. (12) and (27). In order to show that the merging of the two lower Dirac points is actually properly described by the $\mathcal{H}_{+-}$Hamiltonian of Eq. (12), we reconsider the same problem by gapping the upper band from the two lower bands. This is done by adding a dimerization along vertical and horizontal bonds (which breaks the mirror symmetry with respect to the diagonal and the inversion center), as shown in Fig. 7 (top). We introduce a parameter $t^{\prime} \neq 1$ on the $A-B$ bond while the coupling is still 1 on the $B-A$ bond. The Bloch Hamiltonian (basis I) is

$$
H(\mathbf{k})=\left(\begin{array}{ccc}
0 & t^{\prime}+e^{i k_{x}} & t\left(1+e^{i\left(k_{x}+k_{y}\right)}\right) \\
\cdots & 0 & t^{\prime}+e^{i k_{y}} \\
\cdots & \cdots & 0
\end{array}\right) .
$$

We call it the generalized Lieb-kagome model with three hopping amplitudes. This generalization is inspired by the dimerization of the Lieb lattice used in [36] to gap the three bands of the Lieb model. It is also close to, but different from, the anisotropic kagome model discussed in [23].

Using the same procedure as in Sec. III B, we construct the $2 \times 2$ effective Hamiltonian

$$
\begin{aligned}
\mathcal{H}_{M}(\mathbf{q})= & -\left(\frac{t+\delta}{2}+\frac{t^{\prime 2} q_{\perp}^{2}}{2 \delta+6 t}\right) \sigma_{0}+v_{\|} q_{\|} \sigma_{y} \\
& +\left(\frac{3 t-\delta}{2}-\frac{t^{\prime 2} q_{\perp}^{2}}{2 \delta+6 t}\right) \sigma_{z}
\end{aligned}
$$

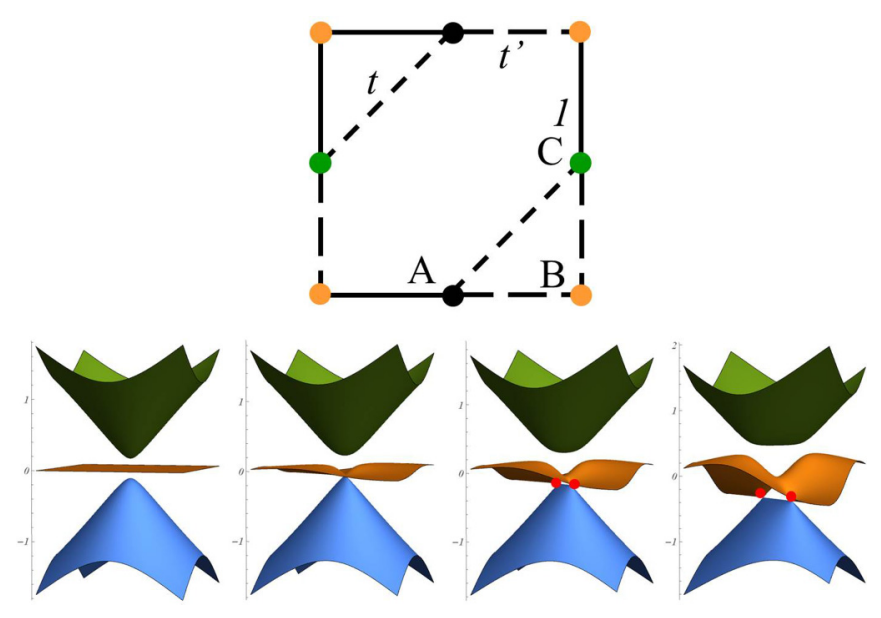

FIG. 7. Top: dimerization of the Lieb-kagome lattice. When $t^{\prime} \neq$ 1, a gap opens between the upper bands. Bottom: closeup energy spectrum at $M$ point, when the upper band has been gapped. The evolution of the lower pair of Dirac points is properly described by an effective $2 \times 2$ Hamiltonian $\mathcal{H}_{+-}$up to their merging. Here, we have chosen $t^{\prime}=0.9, t=0,0.05,0.1,0.2$. The merging transition is reached when $t=\left(1-t^{\prime}\right) / 2$, here when $t=0.05$. Compare with the gapless case on Fig. 4.

with

$$
\delta=\sqrt{t^{2}+\left(1-t^{\prime}\right)^{2}}, \quad \tan \theta=\sqrt{2} \frac{1-t^{\prime}}{t},
$$

and the velocity

$$
v_{\|}=t^{\prime} \cos \frac{\theta}{2}+\sqrt{2} t \sin \frac{\theta}{2} .
$$

The parameters have the following limits:

$$
\begin{gathered}
t^{\prime}=1 \rightarrow \delta=t, \theta=0, v_{\|}=1, \\
t=0 \rightarrow \delta=1-t^{\prime}, \theta=\frac{\pi}{2}, v_{\|}=\frac{t^{\prime}}{\sqrt{2}} .
\end{gathered}
$$

The limit $t \rightarrow 0$ is now well behaved when $t^{\prime} \neq 1$ since the parameter $\delta$ stays finite up to the merging transition which is reached when $\delta=3 t$, that is $t=\left(1-t^{\prime}\right) / 2$. The full evolution is shown on Fig. 7

\section{APPENDIX D: REAL BLOCH HAMILTONIAN AND ORIENTABILITY}

In this Appendix, we study Bloch Hamiltonians that are real and discuss the notion of orientability as defined in Sec. VI B of Ref. [26]. Real Bloch Hamiltonians are possible when the system has both space inversion and time-reversal symmetries. For pedagogical purposes, we start with the two-band staggered Mielke model [25] before turning to the three-band Lieb-kagome model.

We first need to introduce two different Bloch Hamiltonians that can be defined for any tight-binding model possessing more than a single site per unit cell. On the one hand, the Bloch Hamiltonian in the so-called basis I [35] is defined as

$$
H_{\mathrm{I}}(\mathbf{k})=e^{-i \mathbf{k} \cdot \mathbf{R}} H e^{i \mathbf{k} \cdot \mathbf{R}},
$$


where $\mathbf{R}$ is the Bravais lattice position operator. It is periodic with any Bravais lattice vector $\mathbf{G}$ :

$$
H_{\mathrm{I}}(\mathbf{k}+\mathbf{G})=H_{\mathrm{I}}(\mathbf{k}) \text {. }
$$

This basis I is also the convention we choose in the core of the paper when studying the Lieb-kagome model. On the other hand, the Bloch Hamiltonian in the so-called basis II [35] is defined as

$$
H_{\mathrm{II}}(\mathbf{k})=e^{-i \mathbf{k} \cdot \mathbf{r}} H e^{i \mathbf{k} \cdot \mathbf{r}}=e^{-i \mathbf{k} \cdot \delta} H_{\mathrm{I}}(\mathbf{k}) e^{i \mathbf{k} \cdot \delta},
$$

where $\mathbf{r}=\mathbf{R}+\boldsymbol{\delta}$ is the complete position operator involving the Bravais lattice position $\mathbf{R}$ and the intracell position operator $\boldsymbol{\delta}$. For certain Bravais lattice vectors $\mathbf{G}$, it is not periodic but obeys

$$
H_{\mathrm{II}}(\mathbf{k}+\mathbf{G})=e^{-i \mathbf{G} \cdot \delta} H_{\mathrm{II}}(\mathbf{k}) e^{i \mathbf{G} \cdot \delta}
$$

\section{Mielke model with a real Bloch Hamiltonian}

Here, we discuss the case of a two-band real Bloch Hamiltonian, having in mind the staggered Mielke model [25]. This model has inversion and time-reversal symmetries and the inversion center can be chosen onsite. It is defined on the checkerboard lattice. The Bloch Hamiltonian is real and nonperiodic in basis II, whereas it is complex and periodic in basis I (it involves the three Pauli matrices). In basis II, under translation by a Bravais lattice vector $\mathbf{G}$, it behaves as Eq. (D4). When $\mathbf{G}$ is a basis vector of the reciprocal lattice then $e^{i \mathbf{G} \cdot \delta}=\sigma_{z}$. The fact that det $\sigma_{z}=-1$ means that the Bloch Hamiltonian is nonorientable (see Sec. VI B in Ref. [26]). In other words, it is not possible to find a representation in which the Bloch Hamiltonian would be both real and periodic with the first BZ.

The real Bloch Hamiltonian can be written as

$$
\begin{aligned}
H_{\mathrm{II}}(\mathbf{k}) & =h_{x}(\mathbf{k}) \sigma_{x}+h_{z}(\mathbf{k}) \sigma_{z} \\
& =\sqrt{h_{x}^{2}+h_{z}^{2}}\left(\sin \theta \sigma_{x}+\cos \theta \sigma_{z}\right),
\end{aligned}
$$

which defines an angle $\theta(\mathbf{k})$. It produces a checkerboard pattern in reciprocal space with a unit cell twice larger than the first BZ (see the yellow and blue zones in Fig. 8). This is also the case of the pattern of topological charges (winding numbers) as shown by plusses and minuses in the same figure.

Orientability of the Bloch Hamiltonian is related to being able to find a pattern of topological charges which has the periodicity of the first BZ. There is an obstruction to that in the Mielke model (see Fig. 8). Fundamentally, it is this obstruction that allows the evolution of topological charges (i.e., the " $++\rightarrow+-$ " phenomenon).

\section{Lieb-kagome model with a real Bloch Hamiltonian}

As an alternative to the core of the paper, we present here the evolution of the topological charges of band contact points by writing the Bloch Hamiltonian of the Lieb-kagome model in basis II instead of basis I [35], as was done in Eq. (1). In basis II, the Bloch Hamiltonian reads as

$$
H_{\mathrm{II}}(\mathbf{k})=\left(\begin{array}{ccc}
0 & 2 \cos \frac{k_{x}}{2} & 2 t \cos \frac{k_{x}+k_{y}}{2} \\
\ldots & 0 & 2 \cos \frac{k_{y}}{2} \\
\ldots & \ldots & 0
\end{array}\right) .
$$
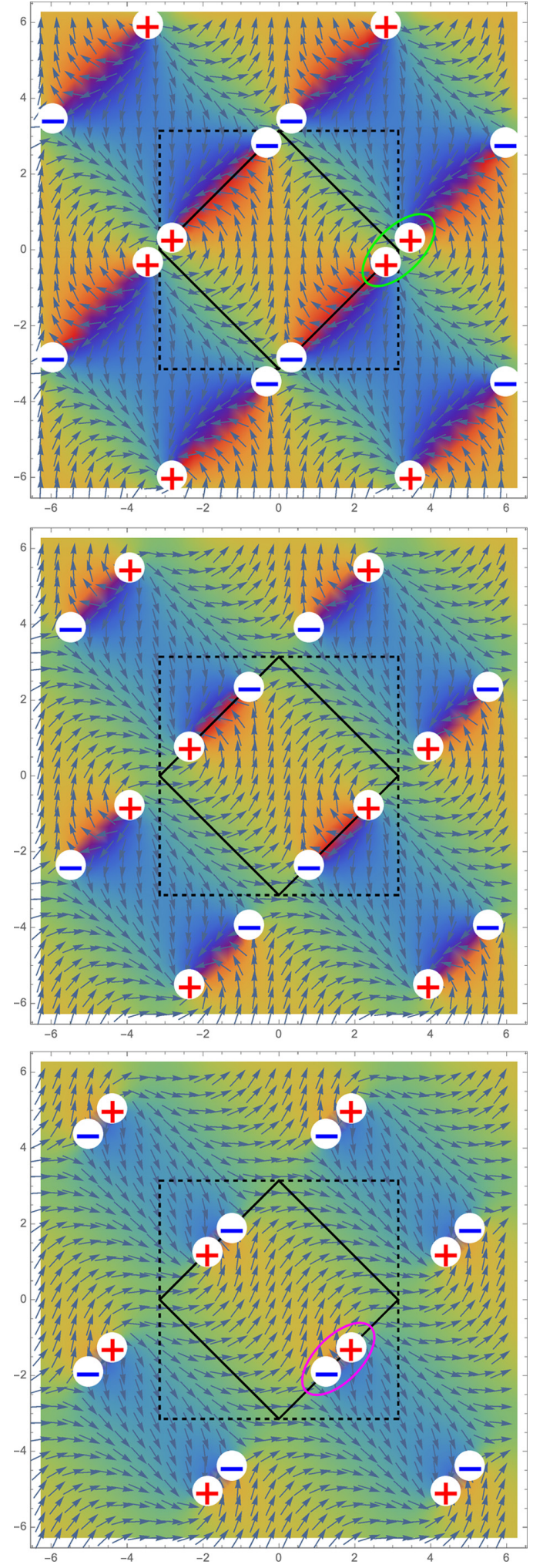

FIG. 8. Pattern of winding numbers (charges shown as red "+" and blue "-") for the staggered Mielke model in reciprocal space. Top: $\delta=0.1$, middle: $\delta=0.5$, and bottom: $\delta=0.9$. The first $\mathrm{BZ}$ is shown in black. The unit cell for the charge pattern is shown as a dashed line (it is twice larger than the first BZ). The arrows and the corresponding color map indicate the angle $\theta(\mathbf{k})$ needed to define the Bloch Hamiltonian in basis II (see text). The unit cell for the Bloch Hamiltonian is the same as for the charge pattern. 


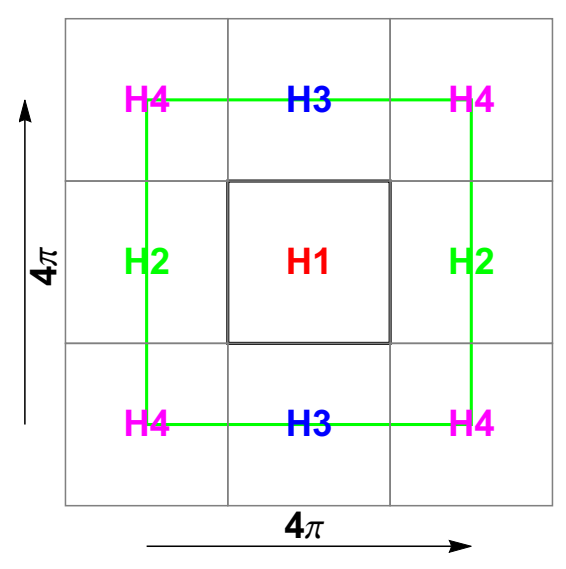

FIG. 9. Pattern of Bloch Hamiltonians for the Lieb-kagome model (in basis II). The first BZ is shown as a thick black square. H1 means $H_{\mathrm{II}}(\mathbf{k}), \mathrm{H} 2=H_{\mathrm{II}}\left(\mathbf{k}+2 \pi \mathbf{u}_{x}\right), \mathrm{H} 3=H_{\mathrm{II}}\left(\mathbf{k}+2 \pi \mathbf{u}_{y}\right)$, and $\mathrm{H} 4$ $=H_{\mathrm{II}}\left(\mathbf{k}+2 \pi \mathbf{u}_{x}+2 \pi \mathbf{u}_{y}\right)$. The unit cell for the Bloch Hamiltonian (shown as a green square) is four times larger than the first BZ.

This Bloch Hamiltonian turns out to be real as a consequence of time-reversal symmetry $H_{\mathrm{II}}(-\mathbf{k})^{*}=H_{\mathrm{II}}(\mathbf{k})$ and inversion symmetry $H_{\mathrm{II}}(-\mathbf{k})=H_{\mathrm{II}}(\mathbf{k})$ with an onsite inversion center. In basis I, space inversion acts in a different way on the Bloch Hamiltonian and does not force it to be real.

In contrast to $H_{\mathrm{I}}(\mathbf{k})$ [see Eq. (1)] that satisfies $H_{\mathrm{I}}\left(k_{x}+\right.$ $\left.2 \pi, k_{y}\right)=H_{\mathrm{I}}\left(k_{x}, k_{y}\right)=H_{\mathrm{I}}\left(k_{x}, k_{y}+2 \pi\right)$, the Bloch Hamiltonian $H_{\mathrm{II}}(\mathbf{k})$ does not have the periodicity of the reciprocal lattice but a doubled periodicity in both $k_{x}$ and $k_{y}$ such that $H_{\mathrm{II}}\left(k_{x}+4 \pi, k_{y}\right)=H_{\mathrm{II}}\left(k_{x}, k_{y}\right)=H_{\mathrm{II}}\left(k_{x}, k_{y}+4 \pi\right)$ and $H_{\mathrm{II}}\left(k_{x}+2 \pi, k_{y}+2 \pi\right)=-H_{\mathrm{II}}\left(k_{x}, k_{y}\right)$. See Fig. 9 .

Because $H_{\mathrm{II}}(\mathbf{k})$ is real, it means that any local two-band Hamiltonian $\mathcal{H}_{\mathrm{II}}(\mathbf{q})$ obtained after projection in the vicinity of a band contact point is also real. Therefore, $\mathcal{H}_{\mathrm{II}}(\mathbf{q})$ can be decomposed onto $\sigma_{x}$ and $\sigma_{z}$ only, so that the corresponding contact point has a winding vector $\vec{w}=w \vec{u}_{y}$ and the notion of winding number $w$ is sufficient. This already means that, in basis II, there is no such thing as a continuous evolution of the winding vector.

We only consider the contact points between the lower two bands. We find that, close to the kagome limit and by expanding in the vicinity of the $\Gamma$ point (similar to Sec. III C), the pair of Dirac point has identical winding numbers (either ++ or -- depending on the basis choice at $\Gamma$ ). In the Lieb limit and by expanding in the vicinity of the $M$ point (similar to Sec. III B), we find that the pair of Dirac points has opposite winding numbers (either +- of -+ depending on the basis chosen at $M$ ). How is this possible? Actually, the Bloch Hamiltonian does not have the periodicity of the reciprocal lattice but a doubled periodicity. It means that the topological charges of the contact points need not have the periodicity of the reciprocal lattice. In other words, the charges attributed to the band contact points within the first BZ are not necessarily the same in "another BZ." Possible patterns respecting the above rules (same charges near $\Gamma$ opposite charges near $M$ ) are shown in red in Fig. 10. The unit cell for the winding numbers is two times larger than the first BZ.

It is not enough to have a Bloch Hamiltonian with enlarged periodicity compared to the $\mathrm{BZ}$ in order to have a pattern
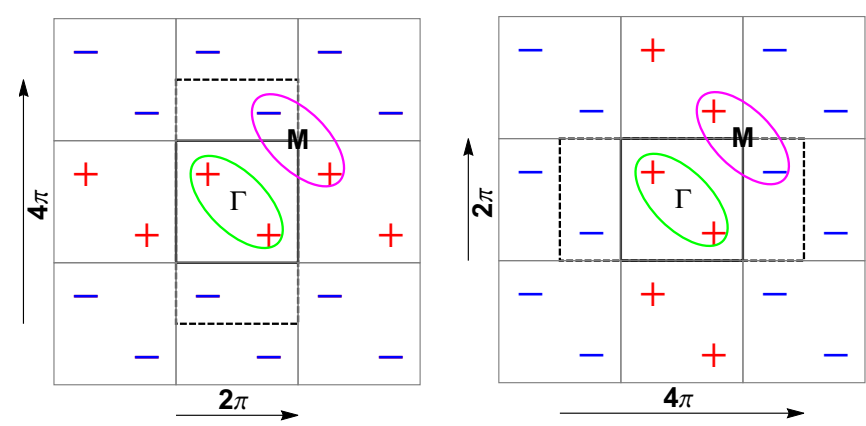

FIG. 10. Pattern of winding numbers (charges shown as red "+" and blue "-") for the band contact points between the lower bands in reciprocal space beyond the first BZ (shown as a thick square) as found using the Bloch Hamiltonian in basis II. The green (respectively magenta) ellipse indicates the ++ (respectively +- ) pattern close to the $\Gamma$ (respectively $M$ ) point. Left: one possible choice, with double periodicity along $k_{y}$. Right: another possibility, with double periodicity along $k_{x}$. The unit cell for the winding numbers (shown as a dashed rectangle) is twice larger than the first BZ.

of topological charges for contact points with an enlarged periodicity. Actually, the periodicity of the Bloch Hamiltonian and of the pattern of topological charges need not be the same [the unit cell in reciprocal space is four times larger than the BZ for the Bloch Hamiltonian (see Fig. 9) and twice larger for the charges (see Fig. 10)]. There is an extra thing that is needed. Ahn et al. have identified it as nonorientability of the real Bloch Hamiltonian [26]. This has to do with the property of a real Bloch Hamiltonian under translation by a reciprocal lattice vector $\mathbf{G}$. If we write $H(\mathbf{k}+\mathbf{G})=O_{\mathbf{G}} H(\mathbf{k}) O_{\mathbf{G}}^{-1}$, with $O_{\mathbf{G}}$ an orthogonal matrix, then if $\operatorname{det} O_{\mathbf{G}}=-1$ the model is said to be nonorientable (and orientable otherwise). For the Lieb-kagome model in basis II and for $\mathbf{G}=2 \pi \mathbf{u}_{x}$, one has

$$
\begin{aligned}
H_{\mathrm{II}}\left(\mathbf{k}+2 \pi \mathbf{u}_{x}\right) & =\left(\begin{array}{ccc}
0 & -2 \cos \frac{k_{x}}{2} & \left.-2 t \cos \frac{k_{x}+k_{y}}{2}\right) \\
\cdots & 0 & 2 \cos \frac{k_{y}}{2} \\
\cdots & \cdots & 0
\end{array}\right) \\
& =O_{x} H_{\mathrm{II}}(\mathbf{k}) O_{x}^{-1},
\end{aligned}
$$

with the orthogonal matrix $O_{x}=\operatorname{diag}(-1,1,1)$. The fact that $\operatorname{det} O_{x}=-1$ means that it is nonorientable. This is also true of the translation by the reciprocal lattice vector $2 \pi \mathbf{u}_{y}$ :

$$
H_{\mathrm{II}}\left(\mathbf{k}+2 \pi \mathbf{u}_{y}\right)=O_{y} H_{\mathrm{II}}(\mathbf{k}) O_{y}^{-1},
$$

with the orthogonal matrix $O_{y}=\operatorname{diag}(1,1,-1)$ such that $\operatorname{det} O_{y}=-1$. However, for the translation by $\mathbf{G}=(2 \pi, 2 \pi)$, we find that

$$
H_{\mathrm{II}}\left(\mathbf{k}+2 \pi \mathbf{u}_{x}+2 \pi \mathbf{u}_{y}\right)=O_{x+y} H_{\mathrm{II}}(\mathbf{k}) O_{x+y}^{-1},
$$

with the orthogonal matrix $O_{x+y}=\operatorname{diag}(-1,1,-1)$ such that $\operatorname{det} O_{x+y}=1$. 
Such an analysis in terms of a real Bloch Hamiltonian does not apply to the case of the generalized Lieb-kagome model of Appendix C. In this case, one has to use the winding vector concept.
[1] G.-B. Jo, J. Guzman, C. K. Thomas, P. Hosur, A. Vishwanath, and D. M. Stamper-Kurn, Ultracold Atoms in a Tunable Optical Kagome Lattice, Phys. Rev. Lett. 108, 045305 (2012).

[2] D. Guzman-Silva, C. Meja-Cortes, M. A. Bandres, M. C. Rechtsman, S. Weimann, S. Nolte, M. Segev, A. Szameit, and R. A. Vicencio, Experimental observation of bulk and edge transport in photonic Lieb lattices, New J. Phys. 16, 063061 (2014).

[3] S. Taie, H. Ozawa, T. Ichinose, T. Nishio, S. Nakajima, and Y. Takahashi, Coherent driving and freezing of bosonic matter wave in an optical Lieb lattice, Sci. Adv. 1, e1500854 (2015).

[4] R. A. Vicencio, C. Cantillano, L. Morales-Inostroza, B. Real, C. Mejía-Cortès, S. Weimann, A. Szameit, and M. I. Molina, Observation of Localized States in Lieb Photonic Lattices, Phys. Rev. Lett. 114, 245503 (2015).

[5] S. Mukherjee, A. Spracklen, D. Choudhury, N. Goldman, P. Öhberg, E. Andersson, and R. R. Thomson, Observation of a Localized Flat-Band State in a Photonic Lieb Lattice, Phys. Rev. Lett. 114, 245504 (2015).

[6] F. Baboux, L. Ge, T. Jacqmin, M. Biondi, E. Galopin, A. Lemaitre, L. LeGratiet, I. Sagnes, S. Schmidt, H. E. Türeci, A. Amo, and J. Bloch, Bosonic Condensation and DisorderInduced Localization in a Flat Band, Phys. Rev. Lett. 116, 066402 (2016).

[7] M. R. Slot, T. S. Gardenier, P. H. Jacobse, G. C. P. van Miert, S. N. Kempkes, S. J. M. Zevenhuizen, C. Morais Smith, D. Vanmaekelbergh, and I. Swart, Experimental realization and characterization of an electronic Lieb lattice, Nat. Phys. 13, 672 (2017).

[8] M. Milićević, G. Montambaux, T. Ozawa, I. Sagnes, A. Lemaître, L. Le Gratiet, A. Harouri, J. Bloch, and A. Amo, Tilted and Type-III Dirac Cones Emerging from Flat Bands in Photonic Orbital Graphene, Phys. Rev. X 9, 031010 (2019).

[9] H. Ozawa, S. Taie, T. Ichinose, and Y. Takahashi, InteractionDriven Shift and Distortion of a Flat Band in an Optical Lieb Lattice, Phys. Rev. Lett. 118, 175301 (2017).

[10] E. Dagotto, E. Fradkin, and A. Moreo, A comment on the Nielsen-Ninomiya theorem, Phys. Lett. 172, 383 (1986).

[11] V. Apaja, M. Hyrkäs, and M. Manninen, Flat bands, Dirac cones, and atom dynamics in an optical lattice, Phys. Rev. A 82, 041402(R) (2010).

[12] N. Goldman, D. F. Urban, and D. Bercioux, Topological phases for fermionic cold atoms on the Lieb lattice, Phys. Rev. A 83, 063601 (2011).

[13] W.-F. Tsai, C. Fang, H. Yao, and J. Hu, Interaction-driven topological and nematic phases on the Lieb lattice, New J. Phys. 17, 055016 (2015).

[14] H. Aoki, M. Ando, and H. Matsumura, Hofstadter butterflies for flat bands, Phys. Rev. B 54, R17296 (1996).

[15] K. Ohgushi, S. Murakami, and N. Nagaosa, Spin anisotropy and quantum Hall effect in the kagomé lattice: Chiral spin state based on a ferromagnet, Phys. Rev. B 62, R6065 (2000).

[16] Y. Xiao, V. Pelletier, P. M. Chaikin, and D. A. Huse, Landau levels in the case of two degenerate coupled bands: kagomé lattice tight-binding spectrum, Phys. Rev. B 67, 104505 (2003).
[17] Y. D. Chong, X.-G. Wen, and M. Soljačić, Effective theory of quadratic degeneracies, Phys. Rev. B 77, 235125 (2008).

[18] K. Sun, H. Yao, E. Fradkin, and S. A. Kivelson, Topological Insulators and Nematic Phases from Spontaneous Symmetry Breaking in 2D Fermi Systems with a Quadratic Band Crossing, Phys. Rev. Lett. 103, 046811 (2009).

[19] B. Dora, I. F. Herbut, and R. Moessner, Occurrence of nematic, topological, and Berry phases when a flat and a parabolic band touch, Phys. Rev. B 90, 045310 (2014).

[20] I. I. Mazin, H. O. Jeschke, F. Lechermann, H. Lee, M. Fink, R. Thomale, and R. Valentí, Theoretical prediction of a strongly correlated Dirac metal, Nat. Commun. 5, 4261 (2014).

[21] L. Ye, M. Kang, J. Liu, F. von Cube, C. R. Wicker, T. Suzuki, C. Jozwiak, A. Bostwick, E. Rotenberg, D. C. Bell, L. Fu, R. Comin, and J. G. Checkelsky, Massive Dirac fermions in a ferromagnetic kagomé metal, Nature (London) 555, 638 (2018).

[22] D. Leykam, A. Andreanov, and S. Flach, Artificial flat band systems: From lattice models to experiments, Adv. Phys. 3, 1473052 (2018).

[23] K. Asano and C. Hotta, Designing Dirac points in twodimensional lattices, Phys. Rev. B 83, 245125 (2011).

[24] W. Jiang, M. Kang, H. Huang, H. Xu, T. Low, and F. Liu, Topological band evolution between Lieb and kagome lattices, Phys. Rev. B 99, 125131 (2019).

[25] G. Montambaux, L.-K. Lim, J.-N. Fuchs, and F. Piéchon, Winding Vector: How to Annihilate Two Dirac Points with the Same Charge, Phys. Rev. Lett. 121, 256402 (2018).

[26] J. Ahn, S. Park, and B.-J. Yang, Failure of Nielsen-Ninomiya Theorem and Fragile Topology in Two-Dimensional Systems with Space-Time Inversion Symmetry: Application to Twisted Bilayer Graphene at Magic Angle, Phys. Rev. X 9, 021013 (2019).

[27] B. Sutherland, Localization of electronic wave functions due to local topology, Phys. Rev. B 34, 5208 (1986).

[28] D. L. Bergman, C. Wu, and L. Balents, Band touching from real-space topology in frustrated hopping models, Phys. Rev. B 78, 125104 (2008).

[29] J. T. Chalker, T. S. Pickles, and P. Shukla, Anderson localization in tight-binding models with flat bands, Phys. Rev. B 82, 104209 (2010).

[30] A. Mielke, Ferromagnetism in the Hubbard model on line graphs and further considerations, J. Phys. A: Math. Gen. 24, 3311 (1991).

[31] G. Volovik, The Universe in a Helium Droplet (Oxford University Press, Oxford, 2009); Exotic Lifshitz transitions in topological materials, Phys. Usp. 61, 89 (2018).

[32] G. Montambaux, F. Piéchon, J.-N. Fuchs, and M. O. Goerbig, Merging of Dirac points in a two-dimensional crystal, Phys. Rev. B 80, 153412 (2009); A universal Hamiltonian for the motion and the merging of Dirac cones in a two-dimensional crystal, Eur. Phys. J. B 72, 509 (2009).

[33] R. de Gail, J.-N. Fuchs, M. O. Goerbig F. Piéchon, and G. Montambaux, Manipulation of Dirac points in graphene-like crystals, Physica B (Amsterdam) 407, 1948 (2012); R. de 
Gail, M. O. Goerbig, and G. Montambaux, Magnetic spectrum of trigonally warped bilayer graphene: Semiclassical analysis, zero modes, and topological winding numbers, Phys. Rev. B 86, 045407 (2012).

[34] P.-O. Löwdin, A note on the quantum-mechanical perturbation theory, J. Chem. Phys. 19, 1396 (1951).
[35] C. Bena and G. Montambaux, Remarks on the tight-binding model of graphene, New J. Phys. 11, 095003 (2009).

[36] C. Poli, H. Schomerus, M. Bellec, U. Kuhl, and F. Mortessagne, Partial chiral symmetry-breaking as a route to spectrally isolated topological defect states in two-dimensional artificial materials, 2D Mater. 4, 025008 (2017). 